\title{
Inhibition of MyD88 Signaling Skews Microglia/Macrophage Polarization and Attenuates Neuronal Apoptosis in the Hippocampus After Status Epilepticus in Mice
}

\author{
Jin-Tao Liu ${ }^{1,2,3}$. Sheng-Xi Wu ${ }^{2}$. Hua Zhang ${ }^{1}$ - Fang Kuang ${ }^{2}$
}

Published online: 15 August 2018

(C) The Author(s) 2018

\begin{abstract}
Inflammation is implicated in epileptogenesis. Activated microglia and macrophages (MG/M $\Phi)$ are found in the brains of patients with epilepsy-related diseases and animal models of epilepsy. It is not yet known how the MG/M $\Phi$ activation phenotype affects pathological changes in the brain after a single seizure. In this study, we had 2 main purposes: first, to characterize poststatus epilepticus (SE) inflammation by tracking MG/M $\Phi$ polarization, and, second, to explore the role of an innate immune receptor adaptor protein, namely, myeloid differentiation primary response gene 88 (MyD88), in the induction of SE in a mouse model. A lithium-pilocarpine model of seizure conditions was generated in C57BL/6 mice. The intensity and distribution of MG/ $\mathrm{M} \Phi$ polarization were tracked by fluorescent immunohistochemistry and Western blotting for the polarization markers inducible nitrogen oxygenized synthase, arginase-1, CD163, and mannose receptor. We observed steadily increasing M1 MG/M $\Phi$ along with MyD88 signal upregulation after SE in the hippocampi of mice, whereas the M2 marker arginase-1 was localized mainly in astrocytes rather than in MG/M $\Phi$. Inhibition or gene knockout of MyD88 reduced M1 MG/M $\Phi$ and gliosis although increasing M2 MG/M $\Phi$ in the hippocampi of SE mice. MyD88 inhibition also augmented glutamate transporter 1 expression and reduced $\mathrm{N}$-methyl-D-aspartate receptor NR1 subunit expression in the hippocampus to protect pyramidal neurons from apoptosis. These data suggest that $\mathrm{MG} / \mathrm{M} \Phi$ polarization after SE impacts the pathological outcome of the hippocampus via MyD88 signaling and point to MyD88 as a potential neuroprotective target for epilepsy therapy.
\end{abstract}

Key Words Microglia/macrophage polarization $\cdot$ Status epilepticus $\cdot$ Hippocampus $\cdot$ MyD88 $\cdot$ Neuroinflammation

Jin-Tao Liu was the main contributor to this work.

Hua Zhang

zhanghua2016@sohu.com

$\triangle$ Fang Kuang

kuangf@fmmu.edu.cn

1 Department of Neurosurgery, Tangdu Hospital, The Fourth Military Medical University, No. 569 Xinsi Road, Xi'an 710038, China

2 Institute of Neurosciences, Department of Neurobiology and Collaborative Innovation Center for Brain Science, The Fourth Military Medical University, No. 169, Changle West Road, Xi'an 710032, China

3 Department of Orthopedics, The 413th Hospital of the Chinese People's Liberation Army, Zhoushan 316000, China

\section{Introduction}

Epilepsy is a central nervous system (CNS) disorder characterized by spontaneous seizures that are caused by abnormal electrical activity of large numbers of neurons in the brain. In patients affected by craniocerebral trauma or intracranial infection, a single seizure can be a harbinger of a gradual increase in seizure probability [1-4]. This apparent facilitation of epileptogenesis may be due to changes in the neuronal microenvironment, such as seizure-induced inflammatory responses and changes in glutamate metabolism $[4,5]$. Recent evidence indicates that, following status epilepticus (SE) events, cerebral glia are involved in CNS inflammatory processes in a manner that produces intense surges in neural $\mathrm{Ca}^{2+}$ conductance via $\mathrm{N}$ methyl-D-aspartate receptor (NMDA) receptors, which then lead to an abnormal increase in intracellular calcium [6-9]. There is a need to clarify putative post-SE pathological glial changes to improve our understanding 
of microenvironment regulation within the brain and, ultimately, to inform the development of treatment strategies for epilepsy.

Among CNS glial cells, microglia (MG) are highly dynamic brain-resident mononuclear macrophages $(\mathrm{M} \Phi)$ that regulate immunological processes such as robust chemotaxis, phagocytosis, and cytokine production. They are known to affect the metabolic activity of neurons and astrocytes under stimulation by pathogens or proinflammatory cytokines [10]. In pathological situations, including epilepsy, circulating $M \Phi$ cross the blood-brain barrier to reinforce activated MG in combating pathogens or injury [11]. Both $\mathrm{M} \Phi$ and $M G$ of systemic origin can be activated in a polarizing manner into extreme pro- or anti-inflammatory states, defined as the M1 and M2 phenotypes, respectively $[10,12]$. The M1 phenotype, which correlates with the Th1 designation and thus represents classical activation, is characterized by the expression of inducible nitric oxide synthase (iNOS), proinflammatory cytokine release, and antigen presentation. The M2 phenotype, which correlates with the Th2 designation and thus represents alternative activation, is characterized by increased production of anti-inflammatory cytokines as well as increased expression of several proteins, including arginase-1 (ARG-1); chitinase-3-like protein (YKL-40), found in inflammatory zone-1 (FIZZ1); CD163; and the mannose receptor (MR). MG polarization has been observed in diverse CNS pathologies, including Alzheimer disease [13, 14], stroke [15], traumatic brain injury [16], and spinal cord injury [17]. The classical M1 phenotype has been associated with neurodegeneration and has a tumor-suppressive role [18], whereas the alternative $\mathrm{M} 2 \mathrm{MG} / \mathrm{M} \Phi$ phenotype has been implicated in neuroprotection and promotion of tissue regeneration [19, 20]. Although some authors question whether MG/M $\Phi$ polarization exists [21], the pro- and anti-inflammatory states of MG/ M $\Phi$ indeed determine the outcomes of many CNS diseases. Therefore, these states may also affect the outcome of epilepsy by regulating $\mathrm{MG} / \mathrm{M} \Phi$ polarization, particularly after seizure.

Seizure is a specific symptom of epilepsy that is caused by synchronized neuron excitation. Neuronal excitotoxicity over a large area produces endogenous damage-associated molecular pattern proteins, such as high mobility group box 1 protein (HMGB-1), which activate MG via TLR4 and other innate immune receptors [7, 22]. The relationship between MG/ $\mathrm{M} \Phi$ polarization and seizure induction has attracted some attention but remains unclear as of yet [23-25]. If $\mathrm{MG} / \mathrm{M} \Phi$ polarization could be documented, regulating $\mathrm{MG} / \mathrm{M} \Phi$ polarization toward anti-inflammation would be a therapeutic strategy to address epilepsy by protecting the CNS environment. Among the ways of regulating MG/M $\Phi$ polarization, the protein myeloid differentiation primary response gene 88 (MyD88), which has been implicated in diverse functions [26], is an interesting candidate for mediating MG/M $\Phi$ involvement in seizure induction. MyD88 is an adaptor protein that is crucial for the proper responsivity of interleukin (IL)-1 and IL-18, as well as for the signaling mechanisms of nearly all Toll-like receptors (TLRs) except TLR3 in MG and M $\Phi$ [22] to mediate the inflammatory response. Notably, we observed previously in rats that blockade of MyD88 early after spinal cord injury protected spinal cord cells from secondary injury [27]. Recent evidence has indicated that MyD88 may influence the direction of MG polarization at the mRNA and protein levels $[28,29]$. Recent studies have documented MyD88 upregulation in epilepsy models, and inhibition of MyD88 significantly suppressed seizure and neuronal apoptosis in animal models [30,31]. However, little is known about whether MG/M $\Phi$ polarization through MyD88 signaling is involved in epileptic seizures. Based on previous studies and our preliminary data, we hypothesized that MyD88 signaling could be used as a target for neuroprotection after seizure by skewing MG/M $\Phi$ toward an anti-inflammatory state.

To test our hypothesis, we designed this study to examine whether MG/M $\Phi$ polarization is involved in SE-induced neuroinflammation and pathological changes in the microenvironment in a lithium-pilocarpine mouse model of epilepsy. We explored the role of MyD88 in post-SE sequelae by examining the hippocampi of MyD88 knockout (KO) mice and mice given intrahippocampal injections of MyD88 inhibitor. Inflammatory processes were followed by fluorescent immunohistochemistry (IHC) and Western blotting experiments across a range of post-SE time points.

\section{Methods and Materials}

\section{Animals and Procedure}

Female C57BL/6 mice (age, 8-10 weeks) were purchased from the Experimental Animal Center of the Fourth Military Medical University, China. MyD88 KO $\left(\mathrm{MyD}^{-/-} 8^{-1}\right.$ mice $(n=6)$ and the corresponding wild-type (WT) C57BL/6 mice $(n=6)$ were purchased from the Model Animal Research Center of Nanjing University. All mice were housed in propylene cages $(33 \mathrm{~cm} \times 18 \mathrm{~cm} \times 14 \mathrm{~cm})$ at an ambient temperature of $22 \pm 2{ }^{\circ} \mathrm{C}$ and relative humidity of $50 \pm 10 \%$. The animals were maintained on a 12-h:12-h light/dark cycle and given ad libitum access to pelleted semipurified mouse chow (Solid, Vital Keao Feed Co., Beijing, China) and filtered tap water. All animal experiments were carried out in accordance with the National Institutes of Health Guide for the Care and Use of Laboratory Animals (NIH Publications No. 80-23, revised 1996). This study was approved by the Institutional Animal Care and Use Committee and the Committee of Animal Use for Research and Education of the Fourth Military Medical University. All efforts were made to minimize animal suffering and reduce the number of animals used. 


\section{Lithium-Pilocarpine SE Model}

A schematic illustration of the epilepsy model is provided in Fig. 1A. Lithium chloride $(480 \mathrm{mg} / \mathrm{kg}, 40 \mathrm{mg} / \mathrm{ml}$,
Sigma, 746460, Saint Louis, MO, USA) was injected intraperitoneally 18 to $19 \mathrm{~h}$ prior to pilocarpine $(96 \mathrm{mg} / \mathrm{kg}$, i.p., $10 \mathrm{mg} / \mathrm{ml}$, Sigma, 1538902, Saint Louis, MO, USA).

The subsequent progression of convulsions was evaluated a

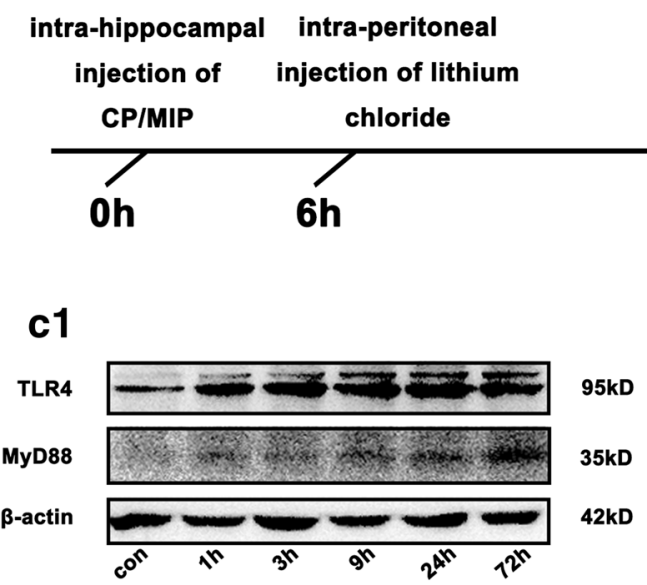

d1

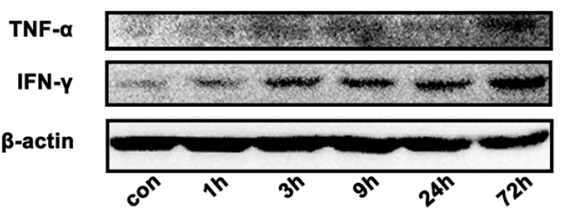

e1

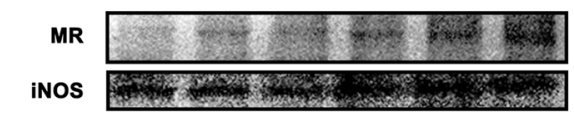

ARG-1

B-actin

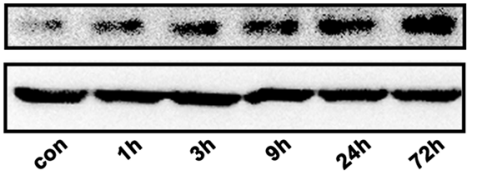

26kD

$25 k D$

42kD
$190 \mathrm{kD}$

$131 \mathrm{kD}$

$35 \mathrm{kD}$

42kD c2

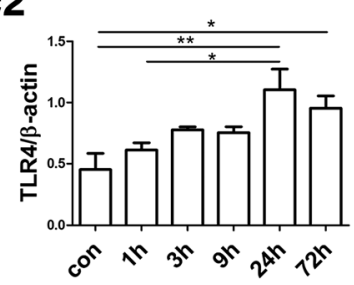

d2

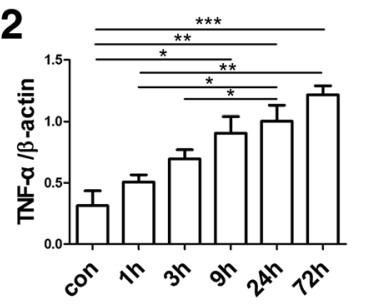

e2

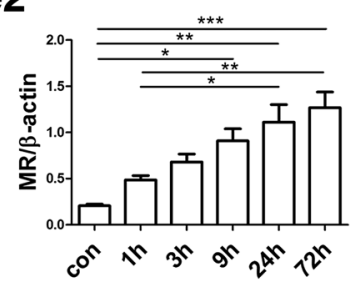

b

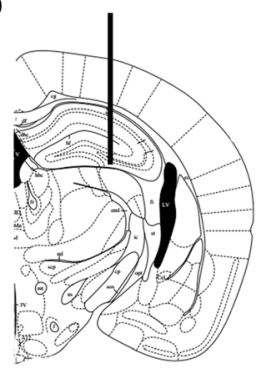

c3

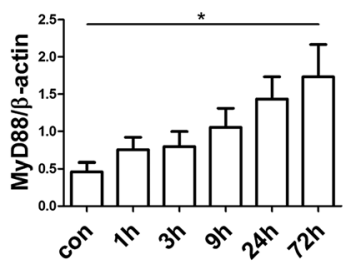

d3

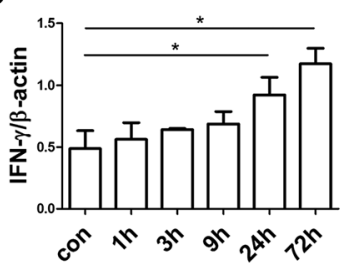

e3

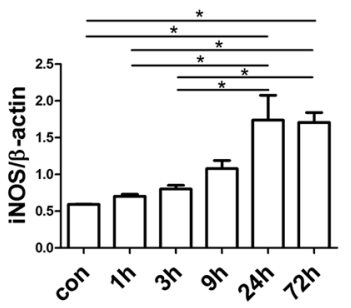

e4

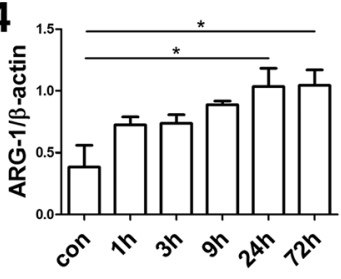

Fig. 1 Experimental design and protein levels of markers for M1/M2 polarization and TLR4-MyD88 signaling in mouse hippocampi across time points in the acute phase after SE. (A) Schematic illustration of the experimental procedure. CP or MIP was injected intrahippocampally $6 \mathrm{~h}$ prior to an intraperitoneal injection of lithium chloride. Twenty-four hours after the intrahippocampal injection, pilocarpine was administered intraperitoneally. SE was halted by diazepam administration. The mice were sacrificed at different time points after SE, and their brains were collected for analysis. (B) Site of stereotactic unilateral hippocampal injection. (C1) Representative immunoblots illustrate that the abundance of TLR4 and

MyD88 in mouse hippocampi increased over time in the acute phase after SE. (C2-C3) Group comparison of TLR4 and MyD88 immunoblots (calibrated relative to $\beta$-actin). (D1) Representative immunoblots showing increases in TNF- $\alpha$ and IFN- $\gamma$ in mouse hippocampi over time up to $72 \mathrm{~h}$ after SE. (D2-D3) Group comparison of TNF- $\alpha$ and IFN- $\gamma$ levels. (E1) Representative immunoblots of MR, iNOS, and ARG-1 for each group; note the relatively rapid increase in iNOS levels over time. (E2-E4) Comparison of MR, iNOS, and ARG-1 levels among the above groups. $N=6$ per group in the Western blots; $* p<0.05, * * p<0.01, * * * p<0.001$ between groups; ANOVA followed by Tukey's test 
according to a modified version of the Racine scale [32] as follows: grades 1 and 2, facial automatisms, tail stiffening, and wetdog shakes; grade 3, low-intensity tonic-clonic seizures marked by unilateral forelimb myoclonus in addition to the grade $1 / 2$ symptoms; grade 4, bilateral forelimb myoclonus and rearing; and grade 5, bilateral forelimb and hindlimb myoclonus and transient loss of postural control. After injection of pilocarpine, the mice's behavior, which consisted generally of salivation, urination, and opisthotonus, was recorded. For this study, the criterion for SE was grade 5 on the aforementioned scale. The first episode of generalized motor convulsions for each mouse was recorded, and those mice that exhibited convulsion of grade 5 were considered successful SE animals. If the first injection of pilocarpine did not induce SE within $30 \mathrm{~min}$, repeated supplemental half-doses of pilocarpine were administered until the mouse reached grade 5 . The number of pilocarpine injections was limited to 3 (total dose $\leq 240 \mathrm{mg} / \mathrm{kg}$ ). SE was not allowed to last longer than $50 \mathrm{~min}$; at the 50-min time point, any ongoing $\mathrm{SE}$ was terminated by administering diazepam $(10 \mathrm{mg} / \mathrm{mg}$, i.p.). In support of the well being of the animals, each mouse was given a daily 2-ml subcutaneous injection of $0.9 \%$ saline containing $1 \%$ glucose for 3 days following SE. The mice in the control group were injected with the same doses of saline and diazepam at the same time points as the corresponding SE groups. Comparisons were performed only within each group.

\section{MyD88 Inhibitory Peptide and Control Peptide Pretreatment}

Prior to lithium chloride administration, animals were anesthetized with $7 \%$ chloral hydrate and mounted on a stereotaxic frame (RWD Life Science, 68867S, Shenzhen, Guangdong, China) for intracerebral injection. A unilateral intrahippocampal stereotactic injection was administered as shown in Fig. 1B. One microliter of $1 \mathrm{mM}$ control peptide (CP) or MyD88 inhibitory peptide (MIP) (NBP2-29328$5 \mathrm{mg}$, Novus, Littleton, CO, USA) was injected unilaterally (left side) into the pyramidal cell layer of the hippocampal CA1 $(2.0 \mathrm{~mm}$ posterior and $1.8 \mathrm{~mm}$ lateral to bregma and $2.3 \mathrm{~mm}$ ventral to the skull surface [33]) with a Hamilton syringe ( $5 \mu$ l, lot No. 87930, Bonaduz, Switzerland). For the sake of preventing backflow, each $1-\mu l$ injection was conducted over a 5-min period, and the injection needle was left in place for an additional $5 \mathrm{~min}$, after which it was withdrawn slowly over another 5-min period. After the injections, the holes in the skulls of the mice were sealed with bone wax. The mice were kept at $37{ }^{\circ} \mathrm{C}$ until they awoke from anesthesia (3 4 h).

\section{Fluorescent IHC}

The successfully generated SE models (grade $5, n=3$ / group) and control animals were sacrificed by transcardial perfusion with $4 \%$ paraformaldehyde in $0.1 \mathrm{M}$ phosphate buffer $(\mathrm{PB})$ for $30 \mathrm{~min}$. Their brains were removed and postfixed in $4 \%$ paraformaldehyde in $\mathrm{PB}$ for $4 \mathrm{~h}$. Subsequently, the brains were equilibrated in $20 \%$ sucrose in PB overnight and then transferred to $30 \%$ sucrose buffer. Coronal sections $(16 \mu \mathrm{m}$ thick) of the forebrain region containing the hippocampus were collected with a freezing microtome and prepared for fluorescent IHC.

Hippocampal sections were immunolabeled to reveal cellular location and changes in HMGB-1, TLR4, MyD88, ARG-1, CD163, iNOS, MR, iba-1, F4/80, glial fibrillary acidic protein (GFAP), and NeuN. After being blocked in 3\% bovine serum albumin (BSA) for $1 \mathrm{~h}$, hippocampal sections were incubated with primary antibody solution containing $0.25 \%$ Triton $\mathrm{X}-100$ and $1 \% \mathrm{BSA}$ at $4{ }^{\circ} \mathrm{C}$ overnight. The following primary antibodies were used: rabbit anti-GFAP (Millipore, AB5804, Darmstadt, Germany, 1:1000), mouse anti-GFAP (Millipore, MAB360, Darmstadt, Germany, 1:1000), rat anti-F4/80 (Affymetrix eBioscience, 144801, Carlsbad, CA, USA, 1:300), rabbit anti-iba-1 (Wako, ctf4377, Osaka, Japan, 1:1000), rabbit anti-NeuN (Millipore, ABN78, Darmstadt, Germany, 1:1000), mouse anti-NeuN (Millipore, MAB377, Darmstadt, Germany, 1:1000), goat anti-ARG-1 (Santa Cruz, sc-18354, Dallas, Texas, USA, 1:200), mouse anti-iNOS (Abcam, ab49999, Cambridge, MA, USA, 1:500), mouse anti-TLR4 (Abcam, ab30667, Cambridge, MA, USA, 1:500), mouse antiMyD88 (R\&D Systems, MAB3109, Minneapolis, MN, USA, $5 \mu \mathrm{g} / \mathrm{ml}$ ), mouse anti-HMGB-1 (Novus, NBP227396, Littleton, CO, USA, $5 \mu \mathrm{g} / \mathrm{ml}$ ), rabbit anti-MR (Abcam, ab64693, Cambridge, MA, USA, 1:500), rabbit anti-GLT-1 (Novus, NBP1-20136, Littleton, CO, USA, $1: 100$ ), and rabbit anti-CD163 (Abcam, ab182422, Cambridge, MA, USA, 1:100). After the primary antibody incubation, sections were washed with $0.01 \mathrm{M}$ phosphate-buffered saline (PBS) 3 times for 5 min each and then incubated with secondary antibody at room temperature for $4 \mathrm{~h}$. After 3 additional PBS washes, the sections were counterstained with Hoechst (Sigma, Saint Louis, MO, USA, 1:5000) for $5 \mathrm{~min}$ and covered with fluorescencepreserving VECTASHIELD mounting medium (Vector, H1000, Shanghai, China). Images were captured with a laser scanning confocal microscope (FV-1000; Olympus, Japan).

\section{Terminal Deoxynucleotidyl Transferase dUTP Nick End Labeling}

For detection of apoptotic cells, 3 fixed mouse brains collected at 3 days after SE were sectioned with a cryostat. The sections were processed according to the instructions of the Cell Death Detection Kit (Roche Molecular Biochemicals, Indianapolis, IN, USA). Images were captured with the same laser scanning confocal microscope introduced above. 


\section{Cell Counting for Fluorescent Labeling}

Images of interest were analyzed with ImageJ and Image Tool software. The density of positively labeled cells in the dentate gyrus (DG), CA1, and CA3 regions of the hippocampus was calculated based on cell counts. For each animal, 3 levels of the hippocampal sections were chosen for each brain, approximately interaural $2.34 \mathrm{~mm}$, bregma $-1.46 \mathrm{~mm}$; interaural $1.86 \mathrm{~mm}$, bregma $-1.94 \mathrm{~mm}$; and interaural $1.34 \mathrm{~mm}$, bregma $-2.46 \mathrm{~mm}$, according to Paxinos and Franklin's mouse brain atlas [33]. The dorsal hippocampus of these levels was divided as CA1, CA3 (including CA2), and DG subareas manually, and the double-stained cells in these levels were judged by eye. Then, the area was evaluated, and the double-labeled cells were counted using Image software. The final cell density was calculated as the number of cells divided by the area.

\section{Western Blot Analysis}

Mice ( $n=6$ at each time point or each group for analysis) were perfused rapidly and systemically with saline, and then their fresh hippocampi were isolated. The fresh hippocampi were frozen quickly in liquid nitrogen $\left(-196{ }^{\circ} \mathrm{C}\right)$. The tissue was treated with RIPA lysis buffer $(1 \mathrm{~g}: 10 \mathrm{ml})$ consisting of $50 \mathrm{mM}$ Tris, $\mathrm{pH} 7.4,150 \mathrm{mM} \mathrm{NaCl}, 5 \mathrm{mM}$ EDTA, $0.1 \%$ SDS, $1 \%$ NP-40, $1 \%$ deoxycholate, $1 \%$ Triton X-100, $10 \mathrm{mM}$ PMSF, $0.1 \%$ proteinase inhibitor, and $0.1 \%$ phosphatase inhibitor. The lysates were incubated in ice for $10 \mathrm{~min}$ and cleared by spinning in a centrifuge (15 min, 12,000 rpm, $4{ }^{\circ} \mathrm{C}$ ). After protein content analysis by the Bradford method, the resultant supernatants were mixed 4:1 with sample buffer and then boiled. Equivalent amounts of protein were loaded and separated in individual lanes of an SDS-PAGE gel and then transferred to a polyvinylidene fluoride membrane. The membranes were blocked with tris-buffered saline with Tween (TBS-T; 5\% low-fat milk, $20 \mathrm{mM}$ Tris-Cl, pH 7.6, $137 \mathrm{mM} \mathrm{NaCl}, 0.1 \%$ Tween 20; for phosphorylated proteins, we used 5\% BSA rather than low-fat milk) for $1 \mathrm{~h}$ at room temperature and then incubated overnight at $4{ }^{\circ} \mathrm{C}$ with the following primary antibodies: rabbit anti-ARG-1 (Sigma, AV45672, Saint Louis, MO, USA, 1:200), rabbit anti-MR (Abcam, ab64693, Cambridge, MA, USA, 1:1000), mouse antiiNOS (Abcam, ab49999, Cambridge, MA, USA, $1: 500)$, rabbit anti-MyD 88 (A b c am, ab2064, Cambridge, MA, USA, 1:500), mouse antiTLR4 (Abgent, AP1504a, San Diego, CA, USA, $1: 500)$, rabbit anti-interferon (IFN)- $\gamma$ (Abcam, ab133566, Cambridge, MA, USA, 1:1000), rabbit anti-tumor necrosis factor (TNF)- $\alpha$ (Abcam, ab6671, Cambridge, MA, USA, 1:200), and rabbit anti- $\beta$-actin (Huabio, R1207-1, Hangzhou, Zhejiang, China, 1:4000). After a wash in TBS-T, the sections were incubated in goat anti-rabbit or goat anti-mouse secondary antibody solutions, as appropriate. After another TBS-T wash, the immunoreactive protein bands were visualized with a chemiluminescence gel imaging system (Aplegen, Pleasanton, CA, USA, Omega Lum G, 81-12100-00, Customizable Omega Lum G Image Capture Software). The immunoblot protein bands were measured by densitometry using Gel-Pro Analyzer software. Western blot bands were analyzed by comparison with an internal control (i.e., $\beta$-actin), and the data are expressed as the means \pm standard errors of the mean (SEM).

\section{Statistical Analyses}

Data from at least 3 animals per group or time point were used in the analyses. The data are presented as the means \pm SEM. When 1-way analysis of variance (ANOVA) showed significant differences among means, pairwise comparisons of the means were performed with Tukey's test. The significance level was set at a $p$ value of less than 0.05 .

\section{Results}

\section{Acute Inflammatory Response to SE Produced Mainly by M1 MG/M $\Phi$ with Astrogliosis}

Hippocampal expression level of inflammatory factors in the acute phase following pilocarpine-induced SE was elevated gradually from hour 1 to hour 72 . Levels of iNOS protein, a key proinflammatory state marker, were dramatically upregulated $24 \mathrm{~h}$ after SE. Meanwhile, the anti-inflammatory state markers ARG-1 and MR protein showed gradual increases. Western blot assays further showed simultaneous activation of TLR4-MyD88 signaling (Fig. 1C-E). MyD88 immunoreactivity was colocalized with that of iba-1, an $\mathrm{MG} / \mathrm{M} \Phi$ marker, throughout the hippocampus, including in the DG, $\mathrm{CA} 1$, and CA3. The number of iba-1/MyD88 doublelabeled cells was increased $(p<0.001)$ over time after SE compared with that in control mice (Fig. 2). Similarly, TLR4 immunoreactivity was also found mainly in iba-1-positive cells, although some other non-MG/ M $\Phi$ cells also expressed TLR4 (Supplementary Fig. 1). We observed a dramatic increase in iNOSimmunopositive $\mathrm{MG} / \mathrm{M} \Phi$ in the hippocampi of mice after SE (Supplementary Fig. 2), whereas MG/M $\Phi$ 
Fig. 2 Representative pictures of MyD88-immunoreactive MG/ $\mathrm{M} \Phi$ and their distribution in the DG, CA1, and CA3 of the hippocampus. (A1-A3) Sections from mice in the control group showed neither activated $\mathrm{MG} / \mathrm{M} \Phi$ nor MyD88-positive cells in these regions. Sections from mice at 1 day (B1-B3) and 3 days (C1C3) after SE showed increased expression of MyD88 specifically in $M G / M \Phi$ (identified by iba-1 immunoreactivity). (D1-D3) Higher magnification of the boxes in (C1-C3). Arrows show cells with strong MyD88 and iba-1 immunoreactivity. The insets of (D1, D2, and D3) show additional high-magnification images of MyD88-positive MG/M $\Phi$. (E) Quantitative analysis of iba-1/ MyD88 double-labeled cells in hippocampi of control group mice 1 or 3 days after SE (means \pm SEM, $n=3) . * p<0.05$, ** $p<$ $0.01, * * * p<0.001$ between groups. One-way ANOVA followed by Tukey's test. ND = not detectable. Scale bars: (A1C3) $100 \mu \mathrm{m}$; (D1-D3) $50 \mu \mathrm{m}$; (D3) (inset) $12.5 \mu \mathrm{m}$

\section{MyD88 ilba-1 Hoechst}
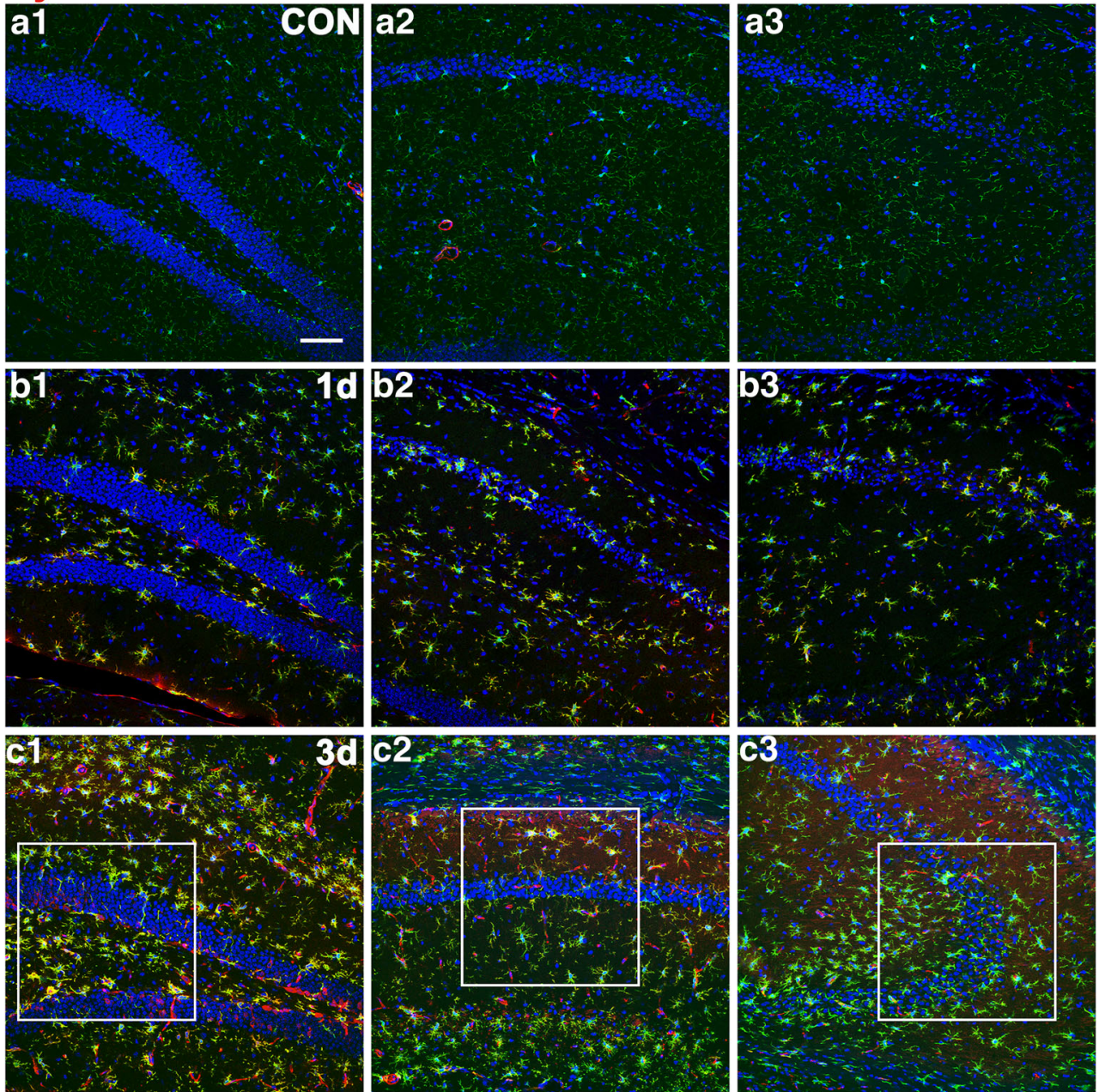

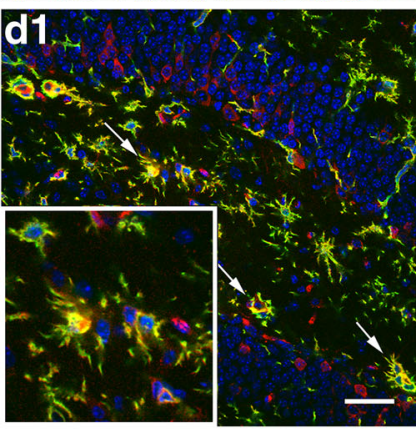

DG

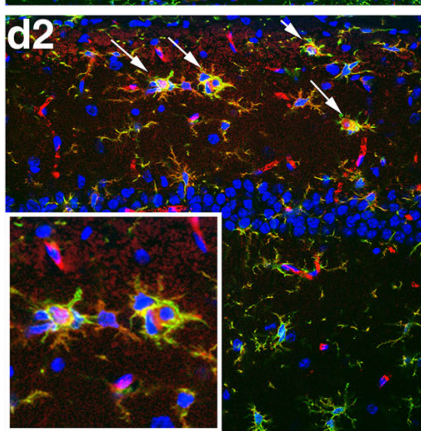

CA1

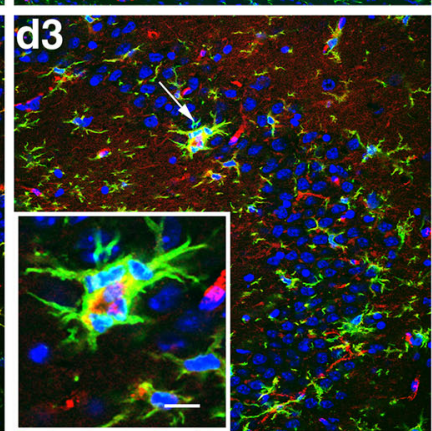

CA3

e

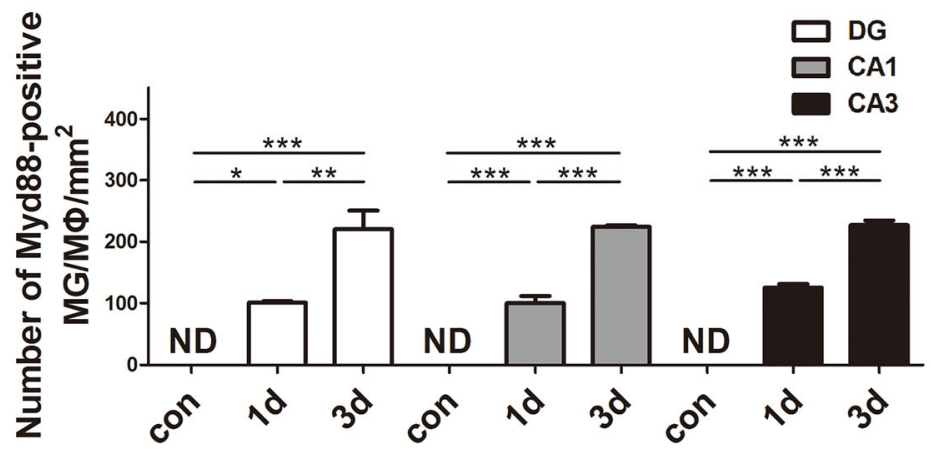


immunopositive for ARG-1 or CD163 were few in number (Supplementary Fig. 3 and Supplementary Fig. 4). Conversely, ARG-1 and MR immunolabeling was found predominantly in astrocytes 1 3 days after SE, indicating that ARG-1 and MR upregulation is mediated primarily by astrocytes rather than $\mathrm{MG} / \mathrm{M} \Phi$
Fig. 3 Hippocampal expression of HMGB-1 at various time points and in different cell types. In control animals, there were hardly any HMGB-1-

immunoreactive cells. HMGB-1 labeling was intense at 14 days after SE and remained to some extent at 28 days. In the acute phase, the HMGB-1-

immunoreactive product was found mainly in the cytoplasm of $\mathrm{MG} / \mathrm{M} \Phi$ and astrocytes. In the chronic phase (28 days), HMGB1 immunoreactivity was also observed in CA1 pyramidal neurons undergoing karyorrhexis. (A1D3) The distribution of HMGB1-positive $\mathrm{MG} / \mathrm{M} \Phi$ in the hippocampi of control animals (A1A3) at 3 days (B1-B3), 14 days (C1-C3), and 28 days (D1-D3) after SE. The inset of (C3) shows strong HMGB-1 labeling in MG/ M $\Phi$. (E1-F3) Colocalization of HMGB-1 immunoreactivity with cell-type markers (NeuN for neurons, GFAP for astrocytes, and iba-1 for $\mathrm{MG} / \mathrm{M} \Phi$ ) in $\mathrm{CA} 1$ at 3 and 28 days. The inset of (F2) shows a higher-magnification view of HMGB-1-labeled karyorrhectic CA1 pyramidal neurons. (A1-B1-C1-D1), HMGB-1 labeling (red); (A2B2-C2-D2), iba-1 labeling (green); (A3-B3-C3-D3) merged images. Scale bars: (A1D3) $200 \mu \mathrm{m}$; (C) (inset) $12.5 \mu \mathrm{m}$; (E1-F3) $50 \mu \mathrm{m}$; (F2) (inset) $12.5 \mu \mathrm{m}$
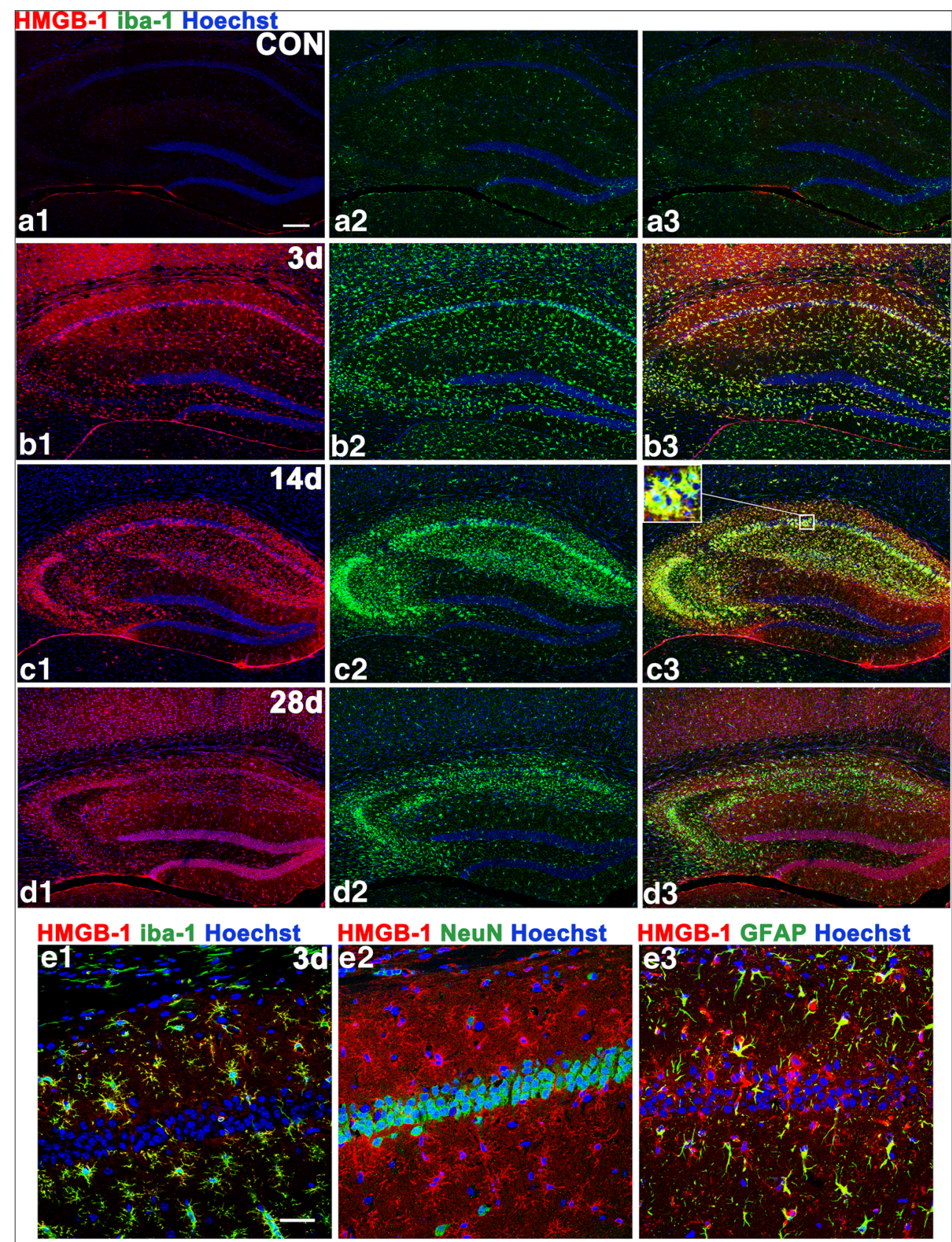

HMGB-1 NeuN Hoechst

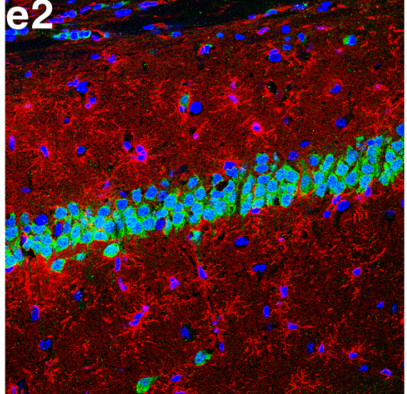

HMGB-1 GFAP Hoechst
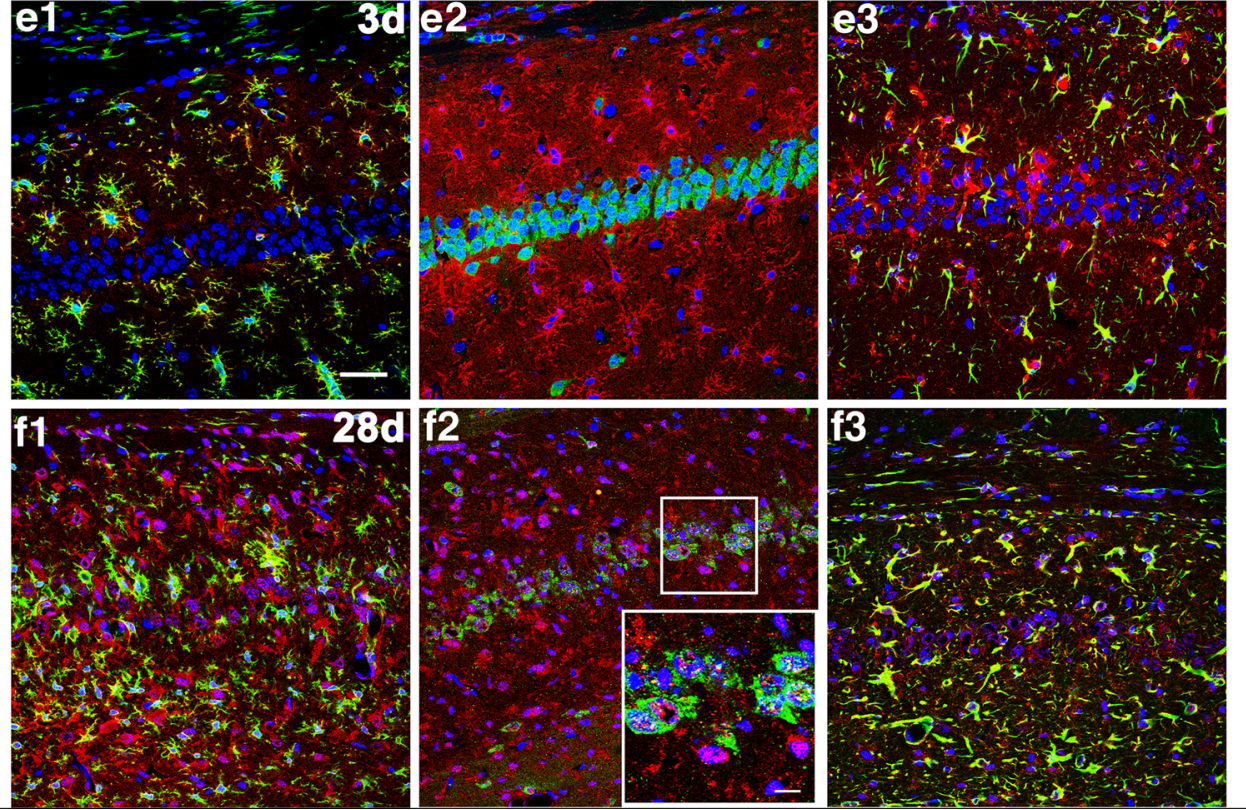
(Supplementary Fig. 3 and Supplementary Fig. 5). MyD88 immunolabeling was observed in iba-1-positive cells but seldom found in astrocytes or neurons (Supplementary Fig. 3). We also found increased numbers of ARG-1immunopositive, activated astrocytes in the hippocampus 3 days after SE (Supplementary Fig. 5).

\section{M1 MG/MФ Response and Astrogliosis Were Aggravated in the Acute to Early Chronic Phases After Pilocarpine-Induced SE}

HMGB-1 immunoreactivity was elevated in activated MG/ M $\Phi 3$ days after SE, remained high 14 days after SE, and
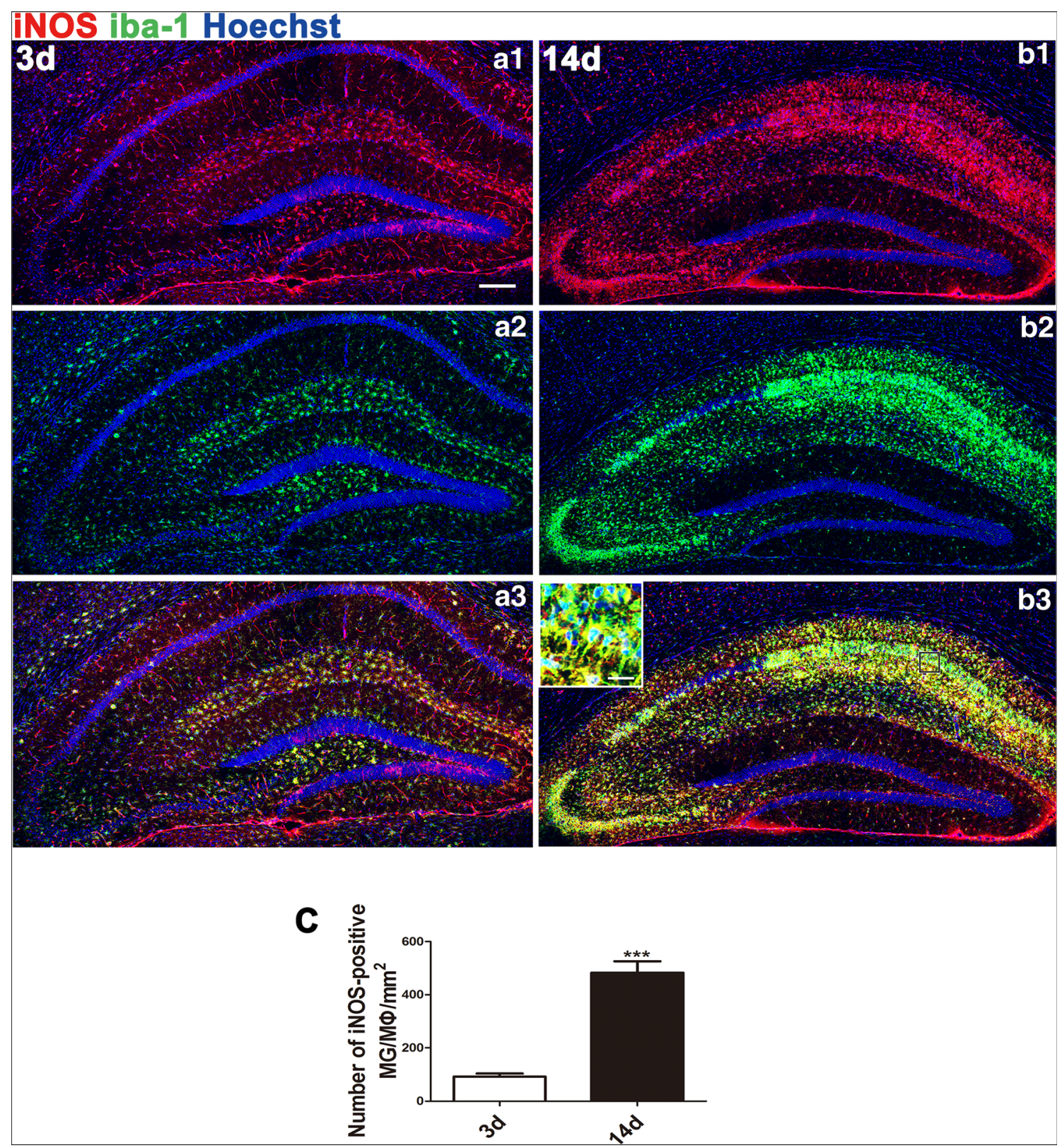

Fig. 4 Distribution of iNOS/iba-1 double-labeled cells in mouse hippocampi 3 and 14 days after SE. Cells that stained positive for both iNOS thalamus. At 14 days, high numbers of $\mathrm{M} 1 \mathrm{MG} / \mathrm{M} \Phi$ were found in areas CA1, CA2, CA3, and CA4 (especially in the stratum pyramidale and stratum radiatum), but $\mathrm{M} 1 \mathrm{MG} / \mathrm{M} \Phi$ were rarely observed in the $\mathrm{DG}$ or outside the hippocampus. The inset in (B3) shows a higher-magnification view of M1 MG/M. . (C) Quantitative analysis of iba-1/iNOS doublelabeled cells in hippocampi at 3 and 14 days after SE (means \pm SEM, $n=$ 3). Independent samples $t$ test; $* * * p<0.001$ between groups. Scale bars: (A1-C) $200 \mu \mathrm{m}$; (B3) (inset) $12.5 \mu \mathrm{m}$ of the CA1 and the hilus of the hippocampus, with some scattered outside the hippocampus, including in the cerebral cortex and the dorsal 

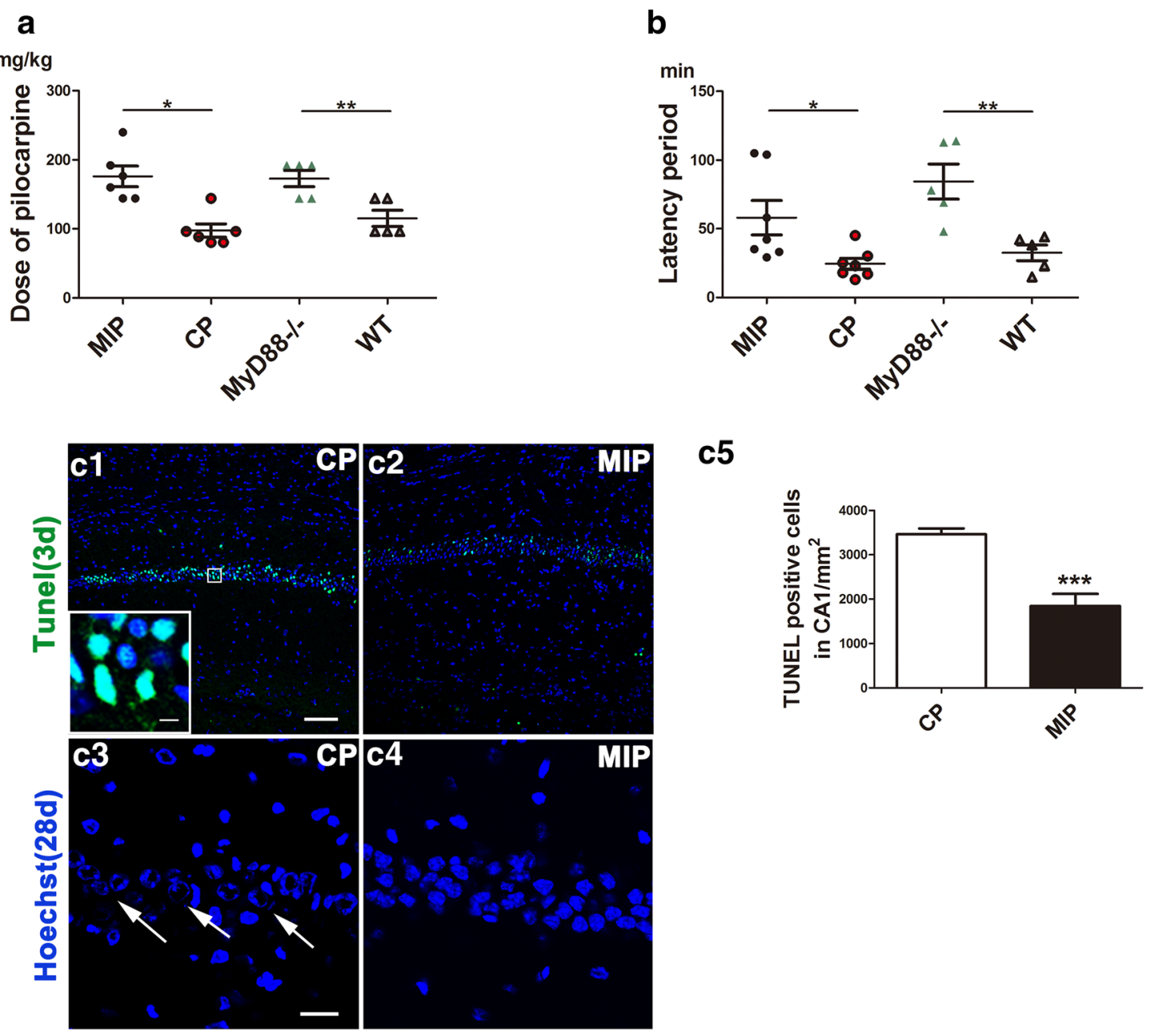

d1

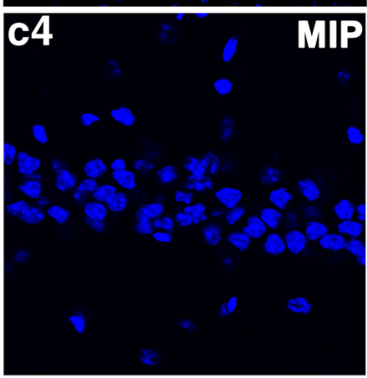

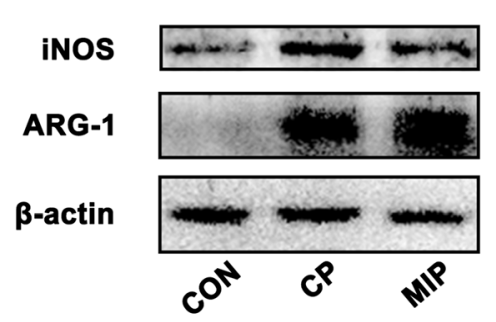

d2

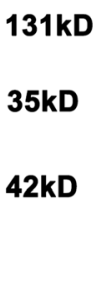

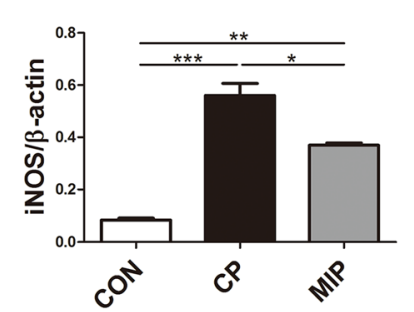

d3

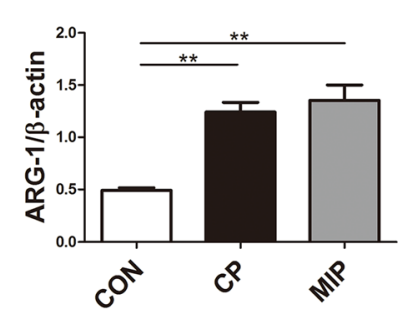

Fig. 5 Effects of inhibition of MyD88 on SE occurrence, pyramidal neuron survival in mouse hippocampi, and expression of $\mathrm{MG} / \mathrm{M} \Phi$ polarization markers. (A) Comparison of the pilocarpine dose necessary to induce SE between the MIP and CP groups and between the MyD88 $8^{-/}$ and WT groups. (B) Comparison of the latency period of SE between the MIP and CP groups and between the MyD $88^{-/-}$and WT groups. Means $\pm \mathrm{SEM} ; n=5 ; * p<0.05$ versus the $\mathrm{CP}$ group/WT group; $* * p<0.01$ versus the $\mathrm{CP}$ group/WT group. Independent samples $t$ tests were performed. TUNEL staining showing CA1 in the CP (C1) and MIP (C2) groups 3 days after SE. The inset in (C1) shows a high-magnification view of TUNEL-positive CA1 pyramidal neurons. (C3) Remarkably

continued to be detected at a lower level in the chronic phase (Fig. 3A-D). Note that on day 3 after SE, HMGB-1 was reduced Hoechst staining in area $\mathrm{CA} 1$ of the hippocampus in the $\mathrm{CP}$ group 28 days after SE. (C4) Hoechst staining showed comparatively normal cell nuclei in the MIP group. Arrows in (C3) indicate cells in karyorrhexis in the CP group. (C5) Quantitative analysis of TUNELstained cells in the pyramidal layer of CA1 3 days after SE between the MIP and CP groups. Independent samples $t$ test, $* * * p<0.001$. Scale bars: (C1, C2) $100 \mu \mathrm{m}$; (C3, C4) $50 \mu \mathrm{m}$; (C1) (inset) $12.5 \mu \mathrm{m}$. (D1) Immunoblots of iNOS and ARG-1 in the control and the CP and MIP groups' hippocampi 3 days after SE. (D2-D3) Group comparison of iNOS and ARG-1 levels calibrated to $\beta$-actin. $* p<0.05$, $* * p<0.01$, $* * * p<0.001$ between groups; 1-way ANOVA followed by Tukey's test

highly expressed in $\mathrm{MG} / \mathrm{M} \Phi$ in particular, with lower expression in astrocytes and hardly any in neurons. By 28 days after 
$\mathrm{SE}$, that is, in the chronic phase, the nuclei of pyramidal neurons in area CA1 were disintegrated but HMGB-1-positive, and more astrocytes were found to be overexpressing HMGB1 at this time than in the acute phase (Fig. 3E-F).

We also observed a significant increase in $\mathrm{M} 1 \mathrm{MG} / \mathrm{M} \Phi$ at 14 days compared with 3 days after SE. At 3 days after SE, M1 MG/M $\Phi$ were concentrated in the stratum lacunosum-moleculare of area CA1 and the hilus of the hippocampus and scattered outside of the hippocampus, particularly in the cerebral cortex and hypothalamus. By 14 days after SE, the distribution of M1 MG/M $\Phi$ changed, becoming most prevalent in areas CA1 (mainly occupying the stratum oriens, the stratum radiatum, and especially the stratum pyramidale), CA2, and CA3 (stratum pyramidale), while remaining rare in the $\mathrm{DG}$ and outside the hippocampus (Fig. 4).

\section{MyD88 Deficiency or Inhibition Blunted SE Induction and Reduced the Number of Apoptotic Neurons and M1 MG/M $\Phi$ but Increased the Number of M2 MG/MФ in the Hippocampus}

To determine whether M1 polarization is MyD88-dependent, we observed the expression of M1 and M2 markers in the brains of MyD88 $8^{-/-}$and MIP-treated mice after SE. During SE induction, we found that MIP-injected and $\mathrm{MyD} 88^{-/-}$mice required a significantly higher dose of pilocarpine and a longer period of time (latency) than WT or CP-injected mice to induce SE (Fig. 5A, B). The mortality during seizure induction was $1 / 6$ for $\mathrm{MyD} 88^{-/-}$mice and 2/6 for WT mice, whereas the mortality for the MIP and CP groups was $6 / 40$ and $10 /$ 40 , respectively.

Terminal deoxynucleotidyl transferase dUTP nick end labeling (TUNEL) staining (3 days after SE) and nuclear staining (28 days after SE) indicated, respectively, that the MIP treatment reduced apoptosis of CA1 neurons in the acute phase and rescued normal nuclear morphology of neurons in the chronic phase (Fig. 5C). Western blots showed lower hippocampal iNOS levels in MIP-injected mice than in CP-injected mice, but iNOS was not lower in the MIP group than in the control group, which had the lowest level of iNOS among the 3 groups. Similarly, the ARG-1 protein level was extremely low in the normal control group but was significantly elevated in the CP group. The ARG-1 level was slightly higher in the MIP group than in the $\mathrm{CP}$ group but not significantly higher in the statistical analysis than in the semiquantitative analysis of immunoblots (Fig. 5D). The number of M1 MG/M $\Phi$ was significantly decreased across the hippocampi of MIP-treated animals compared with the CP group. Typical M1 MG/M $\Phi$ were not observed in MyD88 $8^{-/}$ mice, and few MG/M $\Phi$ expressed only low-intensity iNOS immunopositivity (Fig. 6). At 28 days after SE,
Fig. 6 Effect of MyD88 inhibition and MyD88 deficiency on the numbers and distribution of $\mathrm{M} 1 \mathrm{MG} / \mathrm{M} \Phi$ in the hippocampus 3 days after SE. Immunofluorescence microscopy showed iNOS-positive MG/ $\mathrm{M} \Phi$ in the DG, CA1, and CA3 of mice in the CP group (A1-A3), MIP group (B1-B3), and $\mathrm{MyD} 88^{-/-}$group $(\mathrm{C} 1-\mathrm{C} 3)$ at 3 days. The inset of (A3) shows a high-magnification view of iNOS and iba-1 double-labeled cells (indicated by arrows) in the CA3. The colocalization of iNOS and iba-1 indicated in yellow shows that iNOS labeling appears in nearly the entire $\mathrm{MG} / \mathrm{M} \Phi$, including the processes and somata. (D) Group comparison of the numbers of iNOS/iba-1 double-positive cells in the DG, CA1, and CA3 (means \pm SEM, $n=3$ ). * $p<0.05$, ** $p<0.01$, *** $p<0.001$ between groups; 1-way ANOVA followed by Tukey's test. Scale bars: (A1C3) $100 \mu \mathrm{m}$; (A3) (inset) $12.5 \mu \mathrm{m}$

during the chronic phase, these inhibitory effects of MIP on $\mathrm{M} 1 \mathrm{MG} / \mathrm{M} \Phi$ were more obvious, and the numbers of M1 MG/M $\Phi$ were significantly decreased in all hippocampal subareas of MIP-treated animals compared with those of the CP group (Fig. 7).

Both MyD88 inhibition and deficiency (i.e., KO) induced strong ARG-1 expression, typical of M2 morphology, in MG/ $\mathrm{M} \Phi$ in the acute phase (3 days after SE), and the number of ARG-1 and iba-1 double-labeled cells was significantly increased in the hippocampi of MIP and MyD88 KO animals (Fig. 8). ARG-1-positive MG/M $\Phi$ were still observable throughout the hippocampus in the chronic phase, 28 days after SE, while microgliosis appeared to be suppressed by MIP treatment (Supplementary Fig. 6). MG/M $\Phi$ positive for another M2 marker, CD163, significantly increased in number in the hippocampus of SE mice treated with MIP compared with the mice treated with CP (Supplementary Fig. 4).

\section{Inhibition of MyD88 Reduced NR1 Expression and Increased GLT-1 Expression}

To investigate the potential protective mechanism of MyD88 inhibition, we used Western blots to detect the expression of glutamate receptor/transporter proteins. We found that hippocampal NR1 and NR2b were increased 3 days after SE, whereas NR2a expression was unchanged. Inhibition of MyD88 reduced NR1 expression but did not affect NR2b or NR2a expression. Fluorescent IHC showed that inhibition of MyD88 in the acute phase increased the number of GLT-1positive astrocytes, especially in area CA3. MIP treatment also increased neuronal expression of GLT-1, especially in the neurites of pyramidal neurons (Fig. 9).

\section{Discussion}

In this study, using a pilocarpine mouse model, we observed a progressive exacerbation of inflammation in the hippocampus after SE that was associated mainly with $\mathrm{M} 1 \mathrm{MG} / \mathrm{M} \Phi$ through a MyD88-dependent pathway. Inhibition of MyD88 modulated $\mathrm{MG} / \mathrm{M} \Phi$ polarization, which may in turn suppress 
iNOS iba-1 Hoechst

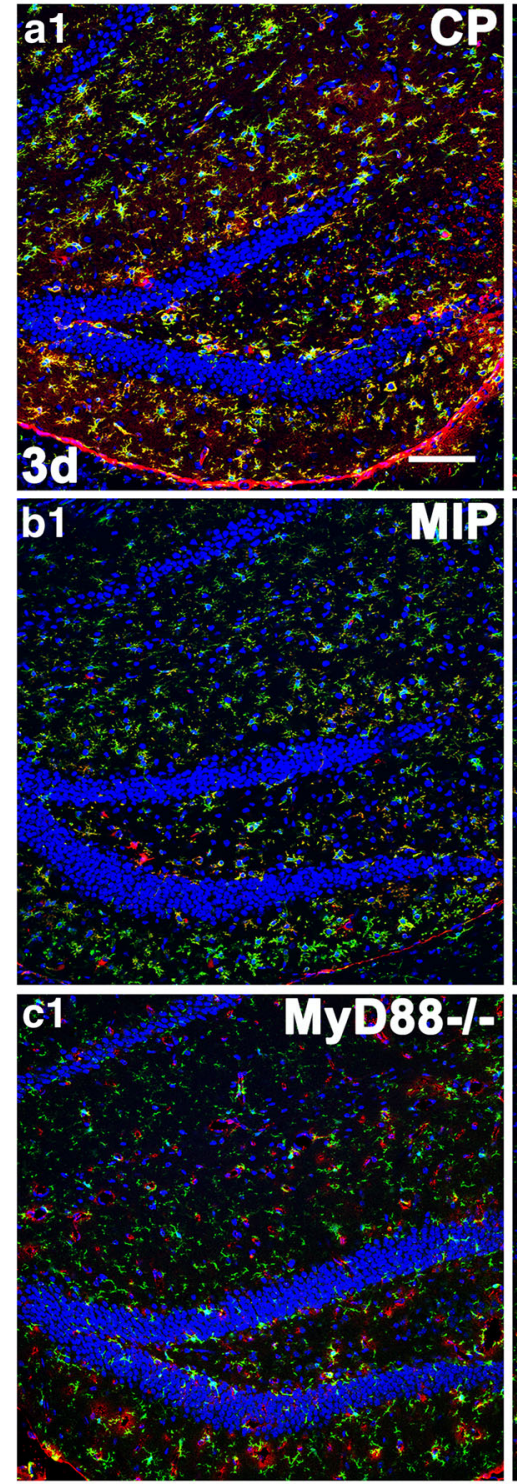

DG
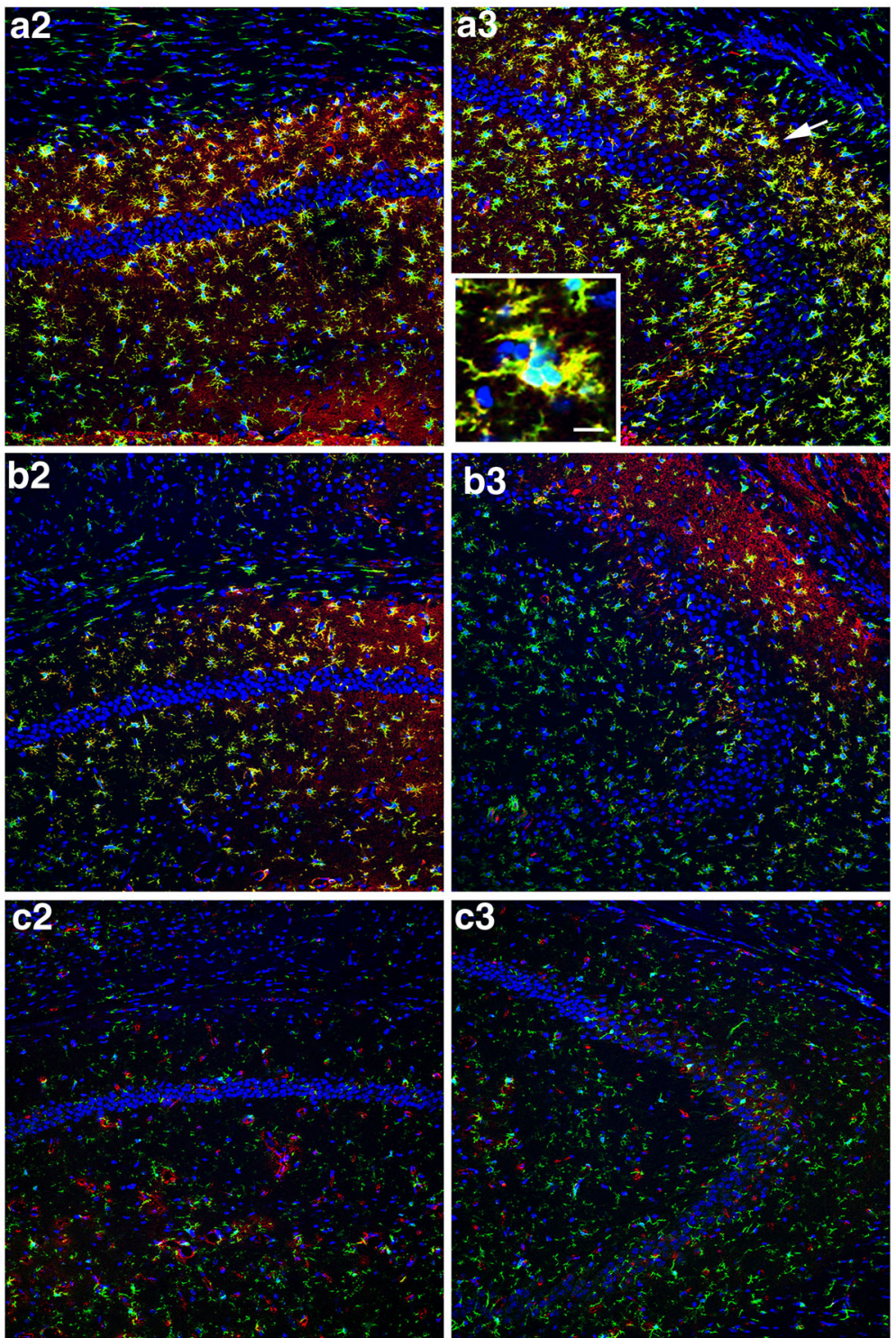

CA1

CA3

d

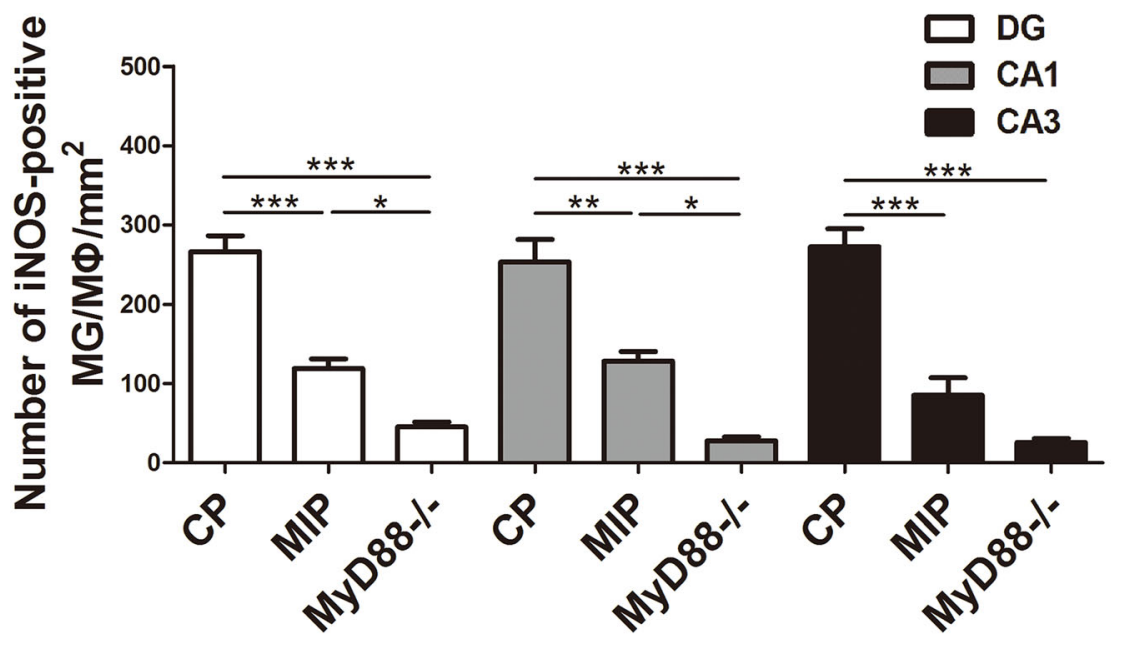




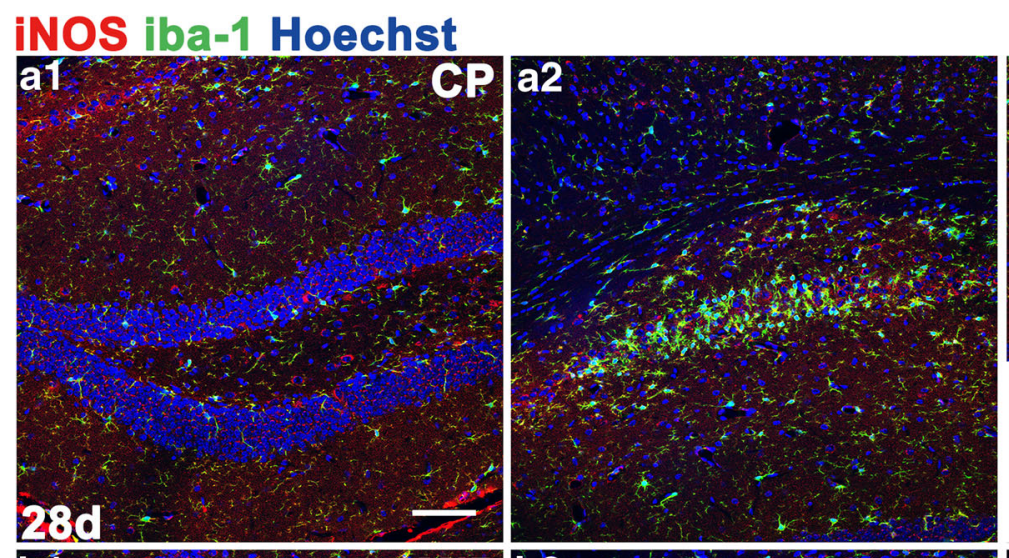

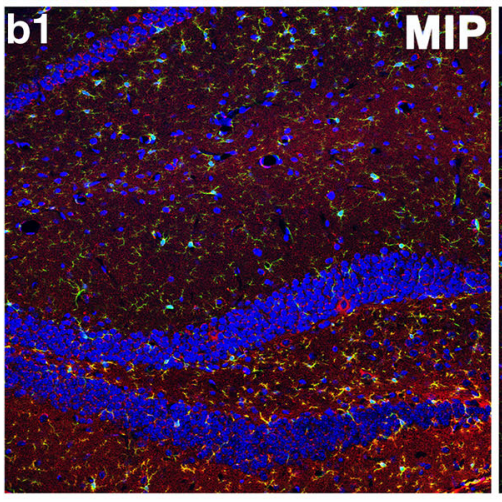

DG

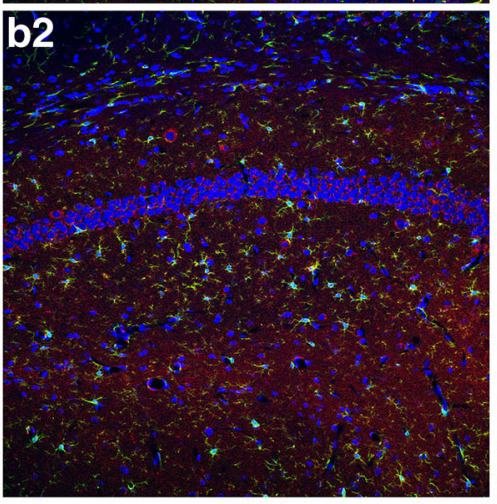

CA1
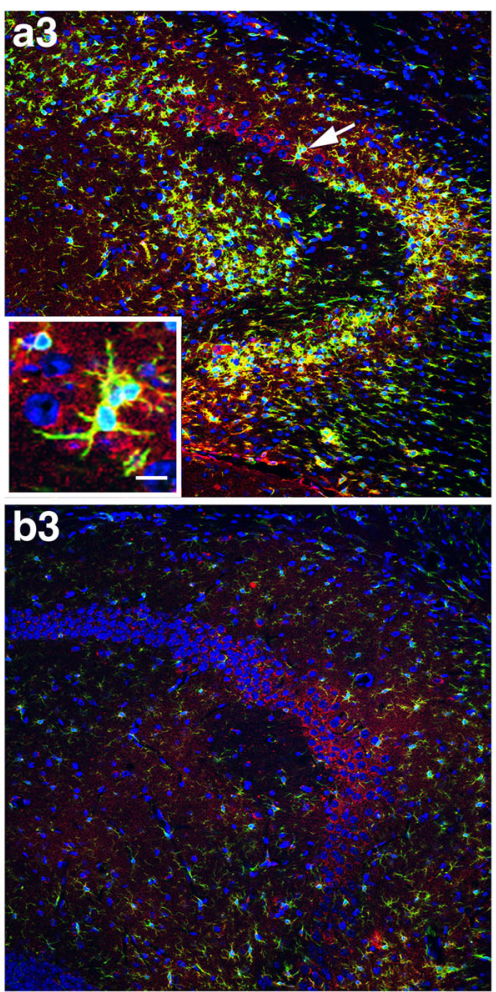

CA3

\section{C}

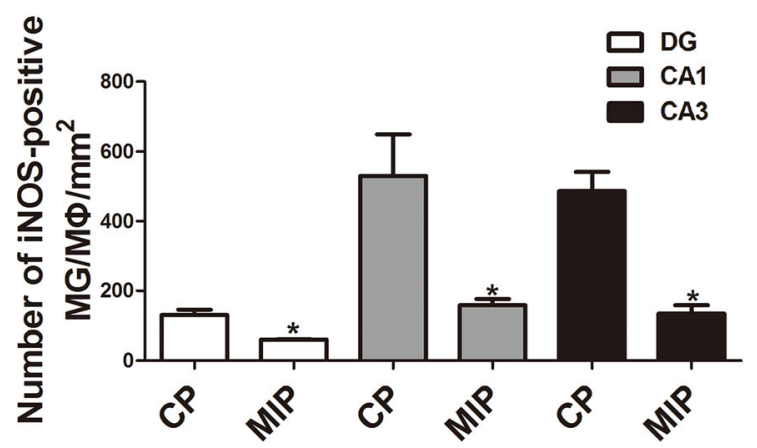

Fig. 7 Effect of inhibition of MyD88 on M1 MG/M $\Phi$ polarization and gliosis in the chronic phase. iNOS-positive $\mathrm{MG} / \mathrm{M} \Phi$ in the CP group (A1-A3) and MIP group (B1-B3) 28 days after SE. The inset of (A3) shows a high magnification of iNOS and iba-1 double-labeled cells in the $\mathrm{CA} 3$ area of the CP group. Note that both iNOS and iba-1 staining could be seen clearly in the processes and soma of the cells. (C) Comparison of

inflammation and gliosis and thus protect hippocampal neurons from apoptosis after SE. We also showed that inhibition of MyD88 upregulated GLT-1 expression and reduced NR1 expression in the hippocampus of SE mice 3 days after SE, which would be expected to improve glutamate transport and reduced postsynaptic excitatory effect. These findings shed light on how MG/M $\Phi$ polarization contributes to the pathological and molecular changes of the microenvironment of the epileptic brain. the numbers of iba-1/iNOS double-positive cells in the DG, CA1, and CA3 between the CP and MIP groups at the 28-day post-SE time point (means $\pm \mathrm{SEM}, n=3$ ). $* p<0.05$ versus the $\mathrm{CP}$ group; $* * p<0.01$ versus the CP group; $* * * p<0.001$ versus the $\mathrm{CP}$ group. Independent samples $t$ tests were performed. Scale bars: (A1-B3) $100 \mu \mathrm{m}$; inset $12.5 \mu \mathrm{m}$

To our knowledge, this study provides the first morphological evidence in the mouse brain documenting MG/M $\Phi$ polarization after SE in an epilepsy model. We provide new evidence on the progression of the neuroinflammation process in the hippocampus after a single SE event. We showed that M1 MG/M $\Phi$ were dramatically increased through the MyD88dependent pathway in the hippocampus after SE and were retained in the chronic phase (28 days after SE). Because $\mathrm{M} 1 \mathrm{MG} / \mathrm{M} \Phi$ produce proinflammatory cytokines that induce 
an increase in excitatory synaptic transmission [34], our findings suggest that M1/M2 phenotype determination could be modulated via MyD88, which may provide a target for supporting microenvironment maintenance in the hippocampus after SE.

\section{Morphological Evidence for M1 MG/MФ Response}

The classical M1 polarization marker iNOS catalyzes NO synthesis. When MG/M $\Phi$ are stimulated by cytokines or microbe components, iNOS is induced to synthesize excess NO, resulting in a series of stress reactions. Recent studies showing that antiepileptic drugs reduce iNOS expression in animals $[35,36]$ suggest that iNOS participates in driving epilepsy.

Here, we demonstrated a continuous increase in iNOSpositive (M1) MG/M $\Phi$ in the hippocampus of mice after SE, and the increase remained predominant in the chronic phase. These data were consistent with a previous report showing sustained inflammation in the hippocampus after SE [37]. Dynamic observation suggested that the distribution of M1 $\mathrm{MG} / \mathrm{M} \Phi$ changed over time. In the acute phase (1 3 days), they were observed mainly in the stratum lacunosum-moleculare of area CA1 and the DG. In this phase, many neurons died of excitotoxicity caused by seizures. These dead cells (including those that died by necrosis and apoptosis) and cell debris induced inflammation via innate immune receptors such as TLR4. In the chronic phase (14 days and beyond), these cells increased in number and were found mainly in the stratum oriens, stratum pyramidale, and stratum radiatum of areas CA1 and CA3, especially the stratum pyramidale. The distribution of M1 MG/M $\Phi$ may reflect the course of neuronal damage. More importantly, the sustained aggregation of M1 MG/M $\Phi$ altered the microenvironment in these areas by way of a hyperinflammatory reaction, leading to apparent apoptosis of pyramidal neurons, as evidenced by TUNEL and Hoechst staining. Here, we do not exclude neuronal necrosis and/or autophagy during this process. These observations suggest that M1 MG/M $\Phi$ cytotoxicity may contribute to hippocampal sclerosis, which is likely involved in the pathogenesis of epilepsy.

These findings also support previous reports indicating in different epilepsy models that microglia M1/M2 polarization plays crucial roles in the pathogenesis of epilepsy[23-25]. Although the details of M1/M2 polarization differ from model to model, all these studies show that M1 polarization is increased in the early stage after SE even before myoclonus symptoms, which is consistent with the findings in our study. M2 polarization has been observed in pilocarpine-induced SE $[23,25]$ and loss-of-function mutations in the cystatin $\mathrm{B}$ gene (CSTB) [24] but not in a kainite-induced SE model [23]. M2 microglia were not found to be predominant after pilocarpineinduced SE in our study, which may be due to variations in model establishment, selected time points, and methods used for identifying M1/M2 phenotypes. Nevertheless, the suggestions of our findings are consistent with the conclusions of these studies that MG/M $\Phi$ polarization after SE crucially affects the outcome of epilepsy through modulation of the neuroinflammatory environment.

In addition to M1/M2 marker expression, expression of the TLR4 ligand HMGB-1 reflects inflammation severity [38, 39]. Along with TLR4 and MyD88, HMGB-1 immunoreactivity was found mainly in MG/M $\Phi$ in the early post-SE inflammatory reaction, indicating that the early inflammatory reaction was mediated mainly by $\mathrm{MG} / \mathrm{M} \Phi$ rather than astrocytes. In the chronic phase, HMGB-1 was highly expressed in karyorrhectic pyramidal neurons, indicating that the inflammatory process after SE is continuous, progressive, and complicated in the chronic phase when multiple cell types are involved. Similarly, in a previous study, changes in inflammation were observed within $3 \mathrm{~h}$ of SE together with HMGB-1 expression in various CA1 cells, and HMGB-1 was observed to act as the TLR4 ligand, thereby playing a key role in the precipitation and recurrence of seizures [6]. Together, these findings suggest that SE-induced HMGB-1 may interact with TLR4 on MG/M $\Phi$ and thereby provoke continuous MG/M $\Phi$ activation in the hippocampus during the acute phase, with inflammation being sustained and becoming complicated and persistent.

\section{Astrocytic ARG-1 Upregulation in the Brains of Mice After SE}

Another glial cell type involved in neuroinflammation is astrocytes. The role of reactive astrocytes in seizures is controversial. They release both pro- and anticonvulsant cytokines $[35,40]$. In addition to participating in the inflammatory response, astrocytes play important roles in glutamate transportation, glutamine synthesis, and water/potassium balance after $\mathrm{SE}$ [41]. Here, we deduce from our finding that astrocytes may dampen the effect of iNOS in the acute phase after SE via upregulation of ARG-1.

ARG-1, which competes with iNOS for the breakdown of arginine $[20,42]$, has an anti-inflammatory and repairfacilitating influence [12]. In our study, Western blotting revealed upregulated ARG-1 expression in the hippocampus during the acute phase of SE. However, our IHC experiments showed that ARG-1 was expressed mainly in astrocytes rather than in $\mathrm{MG} / \mathrm{M} \Phi$ during this period. ARG-1-expressing astrocytes were found previously in an ischemia model as well as in an autoimmune encephalomyelitis model [43, 44], suggesting that ARG-1 in astrocytes may be a reactive response to pathological conditions.

Although ARG-1-positive astrocytes may respond to inflammatory damage after SE, their protective influence is apparently insufficient to overcome the inflammation, given that astrocytes in our model continued to express high levels of HMGB-1, resulting in gliosis. Such post-SE gliosis may 
ARG-1 iba-1 Hoechst
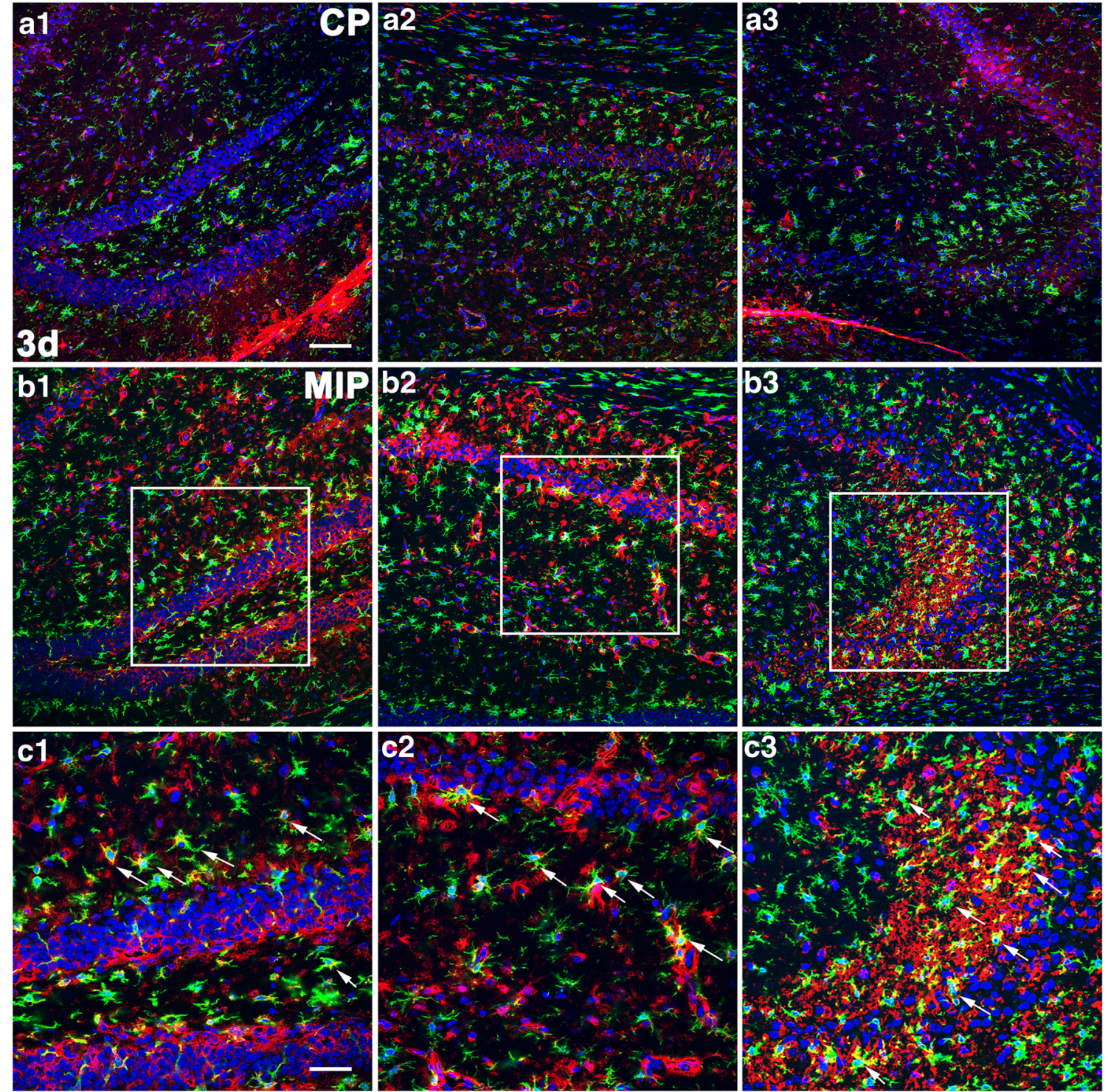

DG

d

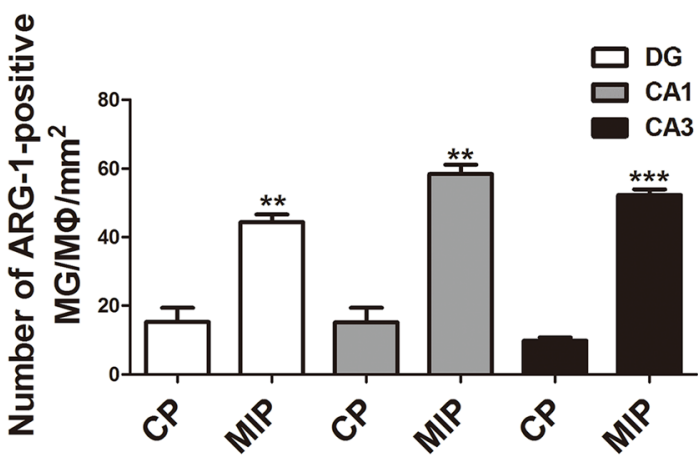

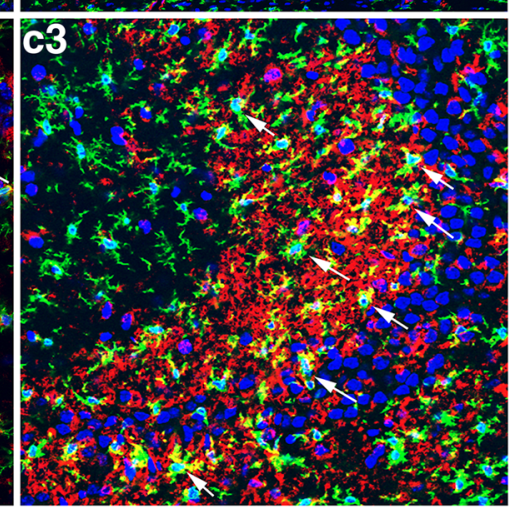

CA3
CA1

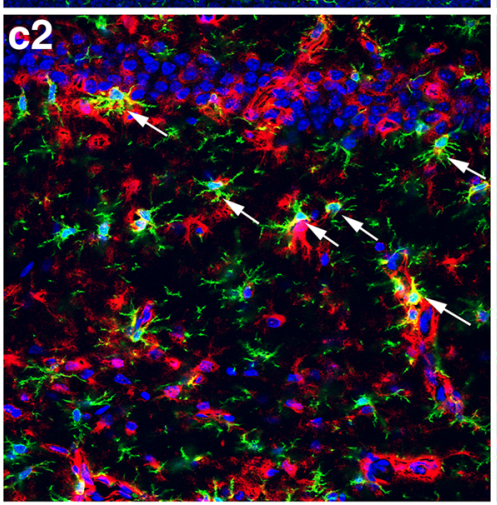

$\mathbf{e}$

\section{ARG-1 iba-1 Hoechst}

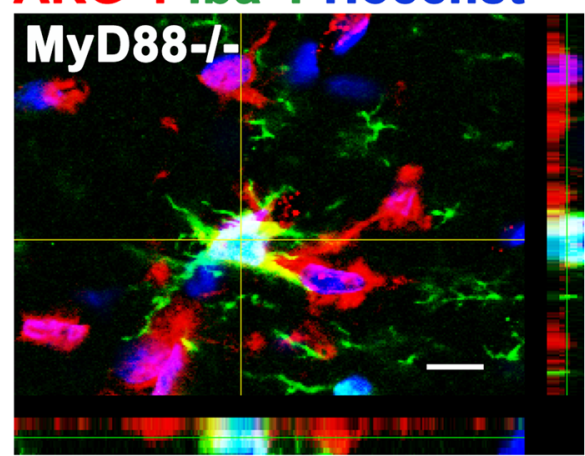

aggravate the complexity of the inflammatory response and, ultimately, may facilitate the development of spontaneous seizures. More studies are needed to distinguish the post-SE stage-specific functions of astrocytes. Notwithstanding, our findings support the notion that the inflammatory environment in the brain may be determined by $\mathrm{MG} / \mathrm{M} \Phi$ polarization. 
Fig. 8 ARG-1 immunolabeled MG/M $\Phi$ in the hippocampi of MyD88inhibited and MyD88-deficient mice 3 days after SE. ARG-1-positive $\mathrm{MG} / \mathrm{M} \Phi$ in the $\mathrm{CP}$ group (A1-A3) and MIP group (B1-B3) at 3 days. (C1-C3) Higher magnification of the boxes in (B1-B3). Arrows indicate colocalization of ARG-1 and iba-1 immunoreactivity. (D) Comparison of the numbers of iba-1/ARG-1 double-positive cells in the DG, CA1, and $\mathrm{CA} 3$ between the CP and MIP groups (means $\pm \mathrm{SEM}, n=3$ ). $* * p<0.01$ versus the $\mathrm{CP}$ group; $* * * p<0.001$ versus the $\mathrm{CP}$ group. Independent samples $t$ tests were performed. (E) Stack scanning of an ARG-1positive $\mathrm{MG} / \mathrm{M} \Phi$ in the hippocampus of an $\mathrm{MyD} 88^{-/-}$mouse showing colocalization of ARG-1 and iba-1 in the cytoplasm. Scale bars: (A1-B3) $100 \mu \mathrm{m}$; (C1-C3) $50 \mu \mathrm{m}$; (E) $12.5 \mu \mathrm{m}$

\section{GFAP GLT-1 Hoechst}
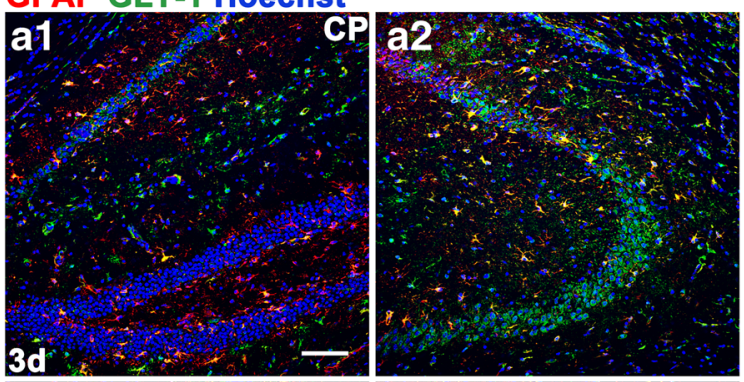

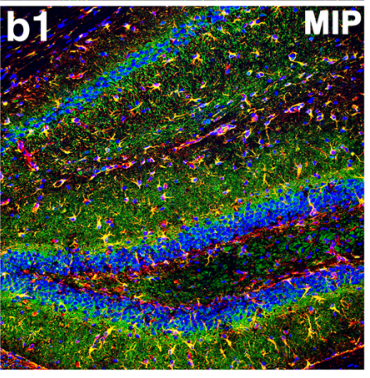

DG

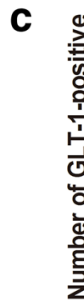

d2

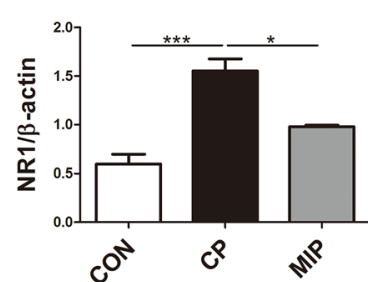

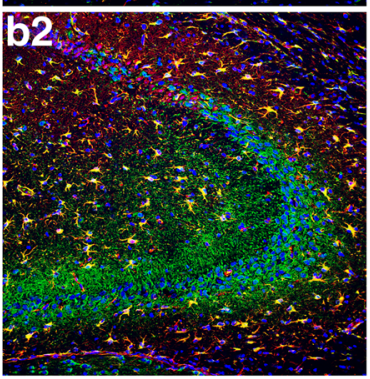

CA3
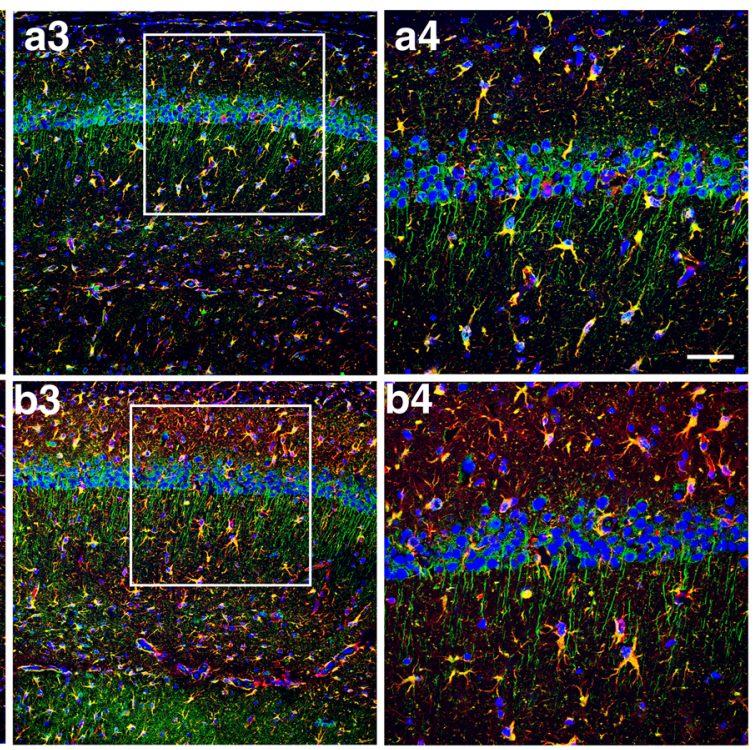

CA1

d1

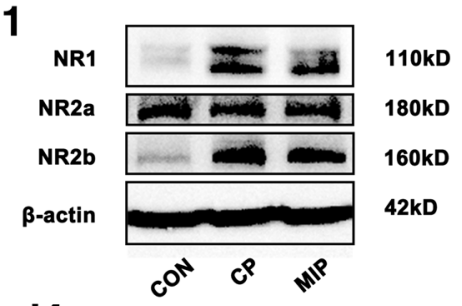

d4

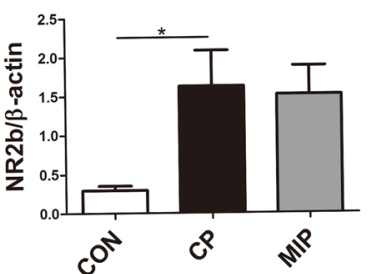

\section{MyD88 Affects SE Outcome by Favoring the M2}

Skewing MG/M $\Phi$ polarization toward M2 could be an option to relieve neuronal excitation-induced inflammation after SE. The MyD88-dependent pathway is triggered by ligand (e.g., amage-associated molecular pattern protein) activation of functions in the brain, including central metabolic processes, cognitive functions, and motor functions [45-47], suggesting

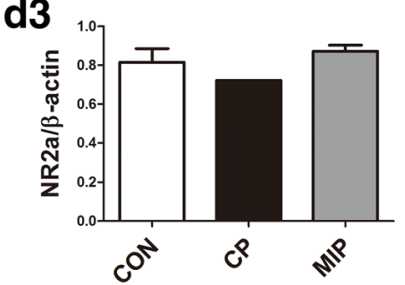

group; $* * p<0.01$ versus the CP group. Independent samples $t$ tests were performed. (D1) Immunoblots of NR1, NR2a, and NR2b for the control, $\mathrm{CP}$, and MIP groups. (D2-D4) Comparison of NR1, NR2a, and NR2b levels among the above groups (calibrated to $\beta$-actin). * $p<0.05$; *** $p<$ 0.001 between groups. One-way ANOVA followed by Tukey's test. Scale bars: (A1-A3, B1-B3) $100 \mu \mathrm{m} ;(\mathrm{A} 4, \mathrm{~B} 4) 50 \mu \mathrm{m}$
Fig. 9 Hippocampal GLT-1 and NR1 expression 3 days after SE with MyD88 inhibition. Sections from the hippocampi of mice in the CP group (A1-A3) and MIP group (B1-B3) 3 days after SE with GLT-1 immunoreactivity in astrocytes and neuronal processes. (A4, B4) Higher magnification of the boxes in (A3) and (B3). (C) Comparison of the numbers of GFAP/GLT-1 double-labeled cells in the DG, CA1, and CA3 between the $\mathrm{CP}$ and MIP groups (means $\pm \mathrm{SEM}, n=3$ ). $* p<0.05$ versus the $\mathrm{CP}$ 
that MyD88 may have extensive regulatory roles in the CNS. Our observation of selective MyD88 expression on MG/M $\Phi$ in the hippocampi of mice after SE indicates a role for MyD88 in $\mathrm{MG} / \mathrm{M} \Phi$ but not in neurons or astrocytes. Inhibition of MyD88 by MIP suppressed M1 polarization and tended to promote M2 polarization, as indicated by a significantly increased number of ARG-1-immunopositive MG/M $\Phi$ in the hippocampi of SE mice. The increase in ARG-1 protein levels in the MIP group was not significant compared with the CP group, as shown by Western blot. This discrepancy may be because astrocytes expressed ARG-1 in response to SE in CPtreated mice. MIP treatment increased the enzyme expression in $\mathrm{MG} / \mathrm{M} \Phi$, but this increase may not dramatically outweigh astrocytic (other cell types cannot be excluded) ARG-1 expression. For CD163, another M2 marker, labeled MG/M $\Phi$ were found to be increased in the hippocampi of MIP-treated SE mice, suggesting M2 polarization by MyD88 inhibition. The inflammatory response was thus downregulated by MyD88 inhibition in the acute phase, which protected hippocampal pyramidal neurons against apoptosis and inhibited gliosis in the chronic phase. These effects account for an improved microenvironment within the hippocampus after SE and thus may lead to relief of hippocampal sclerosis in the chronic phase in the case of epilepsy.

In addition to the tendency toward M2 polarization, MyD88 inhibition or deficiency reduced the occurrence of SE induced by pilocarpine in mice. The data from MyD88 inhibition (MIP-treated mice) are consistent with a previous report on pilocarpine-induced SE in rats [30]. Notably, the blunted occurrence of SE in MyD88 $8^{-/-}$mice relative to WT mice appeared more obvious than that in MIP treatment in comparison with $\mathrm{CP}$ treatment. A previous study on virus infection-induced seizures showed that MyD88 deficiency led to a nonsignificant reduction in the incidence of SE in mice infected with Theiler's murine encephalomyelitis virus [48], whereas the effect of MyD88 deficiency itself on SE was not documented. Accordingly, as we found, genetic MyD88 deficiency was more effective than MIP injection at reducing hippocampal M1 MG/M $\Phi$ responses: $\mathrm{M} 1 \mathrm{MG} / \mathrm{M} \Phi$ were almost entirely absent from the MyD88-deficient hippocampus, where $\mathrm{M} 2 \mathrm{MG} / \mathrm{M} \Phi$ had accumulated, after SE.

MyD88 ablation has also been shown by some [45], but not others [46], to impair locomotor function. This inconsistency may be due to the different behavioral tests used. Regardless, it indicates that the influences of MyD88 deficiency on motor function are probably conditional. In our pilot experiments, we did not observe significant differences in locomotor function between MyD88 inhibition/ablation and control/WT animals either. On the other hand, those behavioral observations did not include convulsions which are specific to seizures. In the present study, the animals' convulsions persisted for $50 \mathrm{~min}$ in SE, which is far different from voluntary, coordinated movements and explorative locomotion, as previously reported. Hence, the blunting of SE by MyD88 inhibition/ ablation may be related to its effects on other responses rather than an influence on movement.

\section{MG/MФ Polarization Affects SE Through Glutamate-Metabolism-Related Excitation of Hippocampal Neurons}

When $\mathrm{MG} / \mathrm{M} \Phi$ polarization was skewed toward M2 via MyD88 inhibition/deficiency, glutamate transportation was also changed in the hippocampi of SE mice. NMDA receptors play critical roles in epileptic excitotoxicity, and NMDA receptor antagonists have been used to treat refractory seizures $[49,50]$. NMDA receptor subunit composition has been reported to be altered in epilepsy models [6, 41-53]. In addition, it has been reported that iNOS acts on mature neurons indirectly, increasing NMDA receptor expression [54]. Increased NMDA receptor expression has also been reported to increase iNOS levels [55], and inhibition of iNOS has been shown to reduce glutamate release [56]. Together, these studies suggest that iNOS enhances the neurotoxic effects of glutamate and thus may form part of a vicious cycle.

Here, we found that $\mathrm{M} 1 \mathrm{MG} / \mathrm{M} \Phi$, along with pyramidal neurons, continuously expressed iNOS in area CA1 and area $\mathrm{CA} 3$ after SE, together with increased expression of glutamate receptor subtypes NR1 and NR2b. Consistent with previous studies $[6,52,53]$, our findings indicate that $\mathrm{M} 1 \mathrm{MG} / \mathrm{M} \Phi$ polarization augments glutamate toxicity, which may lead to apoptosis (and/or other types of cell death) of pyramidal neurons and extensive gliosis, which in turn may underlie, at least in part, the potential occurrence of spontaneous seizures in the chronic phase.

On the other hand, both astrocytic and neuronal GLT-1 have been reported to protect against fatal epilepsy [57, 58], and reduction of glutamate toxicity has been considered to be an effective treatment for epilepsy. We observed that MyD88 inhibition increased both astrocytic and neuronal GLT-1 expression and that GLT-1-positive astrocytes gathered in the DG and in area CA3 during the acute phase. In addition, MyD88 inhibition reduced the expression of NR1, which was upregulated by SE. A similar inhibition of the NMDA receptor was reported in a previous study on SE rats treated with MyD88 inhibitor [30]. These processes may be achieved indirectly by reducing iNOS expression in M1 MG/M $\Phi$. Together, these results suggest that MyD88 inhibition may downregulate glutamate efficiency and transport and may thus reduce glutamate neurotoxicity, thus helping to protect the CNS microenvironment after SE and likely helping to reduce the occurrence of spontaneous seizures.

It should be noted that although MyD88 is expressed mainly in $\mathrm{MG} / \mathrm{M} \Phi$, it is also expressed in other cell types in the CNS, such as neurons [59] and astrocytes. Thus, possible effects of MyD88 inhibition or ablation on other cell types in the present 
study cannot be excluded. Shen and colleagues [60] documented that astrocytic MyD88 ablation reduced LPS-induced neuronal excitatory synaptogenesis in young mice and culture. However, in adults, evidence is lacking on whether MyD88 still works in astrocytes to enhance inflammatory stimulationinduced neuronal excitation. Nevertheless, based on our observation that most of the MyD88 protein expression observed in the hippocampus overlapped with expression of iba-1, an MG/ $\mathrm{M} \Phi$ marker, in the acute phase after SE, the protective effect of MyD88 inhibition on SE animals should be attributed predominantly to alternative $\mathrm{MG} / \mathrm{M} \Phi$ activation in this scenario.

\section{Conclusion}

After SE, augmented M1 polarization of $\mathrm{MG} / \mathrm{M} \Phi$ persists into the chronic phase, and this increase is associated with a deleterious (proinflammatory) microenvironment in the brain. MyD88 inhibition or deficiency is found to skew MG/M $\Phi$ polarization from the M1 to the M2 phenotype and increases the expression of glutamate transporter proteins, eventually improving the pathological outcome and neuronal protection in the hippocampus. Our data suggest that MyD88 may represent a potential target for epilepsy treatment owing to its ability to affect $\mathrm{MG} / \mathrm{M} \Phi$ polarization.

Required Author Forms Disclosure forms provided by the authors are available with the online version of this article.

Abbreviations $A R G-1$ arginase-1, CA Cornu Ammonis, CNS central nervous system, GLT-1 glutamate transporter subtype 1, $H M G B-1$ high mobility group box 1 protein, $I H C$ immunohistochemistry, $I L$ interleukin, iNOS inducible nitric oxide synthase, $M G / M \Phi$ microglia and macrophages, $M R$ mannose receptor, $M y D 88$ myeloid differentiation primary response gene 88, NMDA N-methyl-D-aspartate, $S E$ status epilepticus, $T L E-H S$ temporal lobe epilepsy with hippocampal sclerosis, TLR Tolllike receptor, $W T$ wild type

Authors' Contributions J-TL designed and performed the experiments, analyzed the data, created the figures, and wrote the manuscript. S-XW provided the experimental platform and advice for this study. HZ and FK designed and directed the experiments, analyzed the data, and edited the manuscript.

Funding This work was supported by the National Natural Science Foundation of China (project No. 81671217, 81271433).

\section{Compliance with Ethical Standards}

All animal experiments were carried out in accordance with the National Institutes of Health Guide for the Care and Use of Laboratory Animals (NIH Publications No. 80-23, revised 1996). This study was approved by the Institutional Animal Care and Use Committee and the Committee of Animal Use for Research and Education of the Fourth Military Medical University. All efforts were made to minimize animal suffering and reduce the number of animals used.
Conflict of Interest The authors declare that they have no conflicts of interest.

Open Access This article is distributed under the terms of the Creative Commons Attribution 4.0 International License (http:// creativecommons.org/licenses/by/4.0/), which permits unrestricted use, distribution, and reproduction in any medium, provided you give appropriate credit to the original author(s) and the source, provide a link to the Creative Commons license, and indicate if changes were made.

\section{References}

1. Agrawal A, Timothy J, Pandit L, Manju M. Post-traumatic epilepsy: an overview. Clin Neurol Neurosurg 2006;108: 433-439.

2. Kim LG, Johnson TL, Marson AG, Chadwick, DW. Prediction of risk of seizure recurrence after a single seizure and early epilepsy: further results from the MESS trial. Lancet Neurol 2006;5: 317-322.

3. Chiu SS, Catherine YC, Lau YL, Peiris M. Influenza A infection is an important cause of febrile seizures. Pediatrics 2001;108: e63-e63.

4. Vezzani A, Fujinami RS, White HS, Preux PM, Blümcke I, Sander JW, Löscher W. Infections, inflammation and epilepsy. Acta Neuropathol 2016;131: 211-234.

5. Çavuş I, Romanyshyn JC, Kennard JT, Farooque P, Williamson A, Eid T, Spencer SS, Duckrow R, Dziura J, Spencer DD. Elevated basal glutamate and unchanged glutamine and gaba in refractory epilepsy. Ann Neurol 2016;80: 35-45.

6. Maroso M, Balosso S, Ravizza T, Liu J, Aronica E, Iyer AM, Rossetti C, Molteni M, Casalgrandi M, Manfredi AA, Bianchi ME, Vezzani A. Toll-like receptor 4 and high-mobility group box1 are involved in ictogenesis and can be targeted to reduce seizures. Nat Med 2010;16: 413-419.

7. Vezzani A, Maroso M, Balosso S, Sanchez MA, Bartfai T. IL-1 receptor/Toll-like receptor signaling in infection, inflammation, stress and neurodegeneration couples hyperexcitability and seizures. Brain Behav Immun 2011;25: 1281-1289.

8. Devinsky O, Vezzani A, Najjar S, De Lanerolle NC, Rogawski MA. Glia and epilepsy: excitability and inflammation. Trends Neurosci 2013;36: 174-184.

9. Vezzani A, Aronica E, Mazarati A, Pittman QJ. Epilepsy and brain inflammation. Exp Neurol 2013;244: 11-21.

10. Orihuela R, McPherson CA, Harry GJ. Microglial M1/M2 polarization and metabolic states. Brit J Pharmacol 2016;173: 649-665.

11. Vezzani A, Granata T. Brain inflammation in epilepsy: experimental and clinical evidence. Epilepsia 2005;46: 1724-1743.

12. $\mathrm{Hu} \mathrm{X}$, Leak RK, Shi Y, Suenaga J, Gao Y, Zheng P, Chen J. Microglial and macrophage polarization - new prospects for brain repair. Nat Rev Neurol 2015;11: 56-64.

13. Mandrekar-Colucci S, Karlo JC, Landreth GE. Mechanisms underlying the rapid peroxisome proliferator-activated receptor- $\gamma$-mediated amyloid clearance and reversal of cognitive deficits in a murine model of Alzheimer's disease. J Neurosci 2012;32: 10117-10128.

14. Moehle MS, West AB. M1 and M2 immune activation in Parkinson's disease: foe and ally? Neuroscience 2015;302: 59-73.

15. Hu X, Li P, Guo Y, Wang H, Leak RK, Chen S, Gao Y, Chen J. Microglia/macrophage polarization dynamics reveal novel mechanism of injury expansion after focal cerebral ischemia. Stroke 2012;43: 3063-3070.

16. Wang G, Zhang J, Hu X, Zhang L, Mao L, Jiang X, Liou AK, Leak RK, Gao Y, Chen J. Microglia/macrophage polarization dynamics in white matter after traumatic brain injury. J Cereb Blood Flow Metab 2013;33: 1864-1874.

17. Kigerl KA, Gensel JC, Ankeny DP, Alexander JK, Donnelly DJ, Popovich PG. Identification of two distinct macrophage subsets with divergent effects causing either neurotoxicity or regeneration in the injured mouse spinal cord. J Neurosci 2009;29: 13435-13444. 
18. Mantovani A, Allavena P. The interaction of anticancer therapies with tumor-associated macrophages. J Exp Med 2015;212: 435-445.

19. Ma TC, Campana A, Lange PS, Lee HH, Banerjee K, Bryson JB, Mahishi L, Alam S, Giger RJ, Barnes S, Morris, SM, Willis DE, Twiss JL, Filbin MT, Ratan RR. A large-scale chemical screen for regulators of the arginase 1 promoter identifies the soy isoflavone daidzein as a clinically approved small molecule that can promote neuronal protection or regeneration via a cAMP-independent pathway. J Neurosci 2010;30: 739-748.

20. Caldwell RB, Toque HA, Narayanan SP, Caldwell RW. Arginase: an old enzyme with new tricks. Trends Pharmacol Sci 2015;36: 395-405.

21. Ransohoff RM. A polarizing question: do M1 and M2 microglia exist? Nat Neurosci 2016;19: 987-991.

22. Chen H, Jiang Z. The essential adaptors of innate immune signaling. Protein Cell 2013;4: 27-39.

23. Benson MJ, Manzanero S, Borges K. Complex alterations in microglial M1/M2 markers during the development of epilepsy in two mouse models. Epilepsia 2015;56: 895-905.

24. Okuneva O, Körber I, Li Z, Tian L, Joensuu T, Kopra O Lehesjoki AE. Abnormal microglial activation in the $\mathrm{Cstb}^{-} /-$mouse, a model for progressive myoclonus epilepsy, EPM1. Glia 2015;63(3), 400-411.

25. Li T, Zhai X, Jiang J, Song X, Han W, Ma J, Xie L, Cheng L, Chen $\mathrm{H}$, Jiang L. Intraperitoneal injection of IL-4/IFN- $\gamma$ modulates the proportions of microglial phenotypes and improves epilepsy outcomes in a pilocarpine model of acquired epilepsy. Brain Res 2017; 1657:120-129

26. Kaisho T, Akira S. Dendritic-cell function in Toll-like receptor- and MyD88-knockout mice. Trends Immunol 2001;22: 78-83.

27. Yao A, Jia L, Zhang Y, Ma QR, Cheng P, Liu L, Ju G, Kuang F. Early blockade of TLRs MyD88-dependent pathway may reduce secondary spinal cord injury in the rats. Evid Based Complement Alternat Med 2012

28. Hayakawa K, Okazaki R, Morioka K, Nakamura K, Tanaka S, Ogata T. Lipopolysaccharide preconditioning facilitates M2 activation of resident microglia after spinal cord injury. J Neurosci Res 2014;92: 1647-1658.

29. Aikawa T, Mogushi K, Iijima-Tsutsui K, Ishikawa K, Sakurai M, Tanaka H, Mizusawa H, Watase K. Loss of MyD88 alters neuroinflammatory response and attenuates early Purkinje cell loss in a spinocerebellar ataxia type 6 mouse model. Hum Mol Genet 2015; 24: 4780-4791.

30. Wang N, Han X, Liu H, Zhao T, Li J, Feng Y, Mi X, Zhang Y, Chen $\mathrm{Y}$, Wang X. Myeloid differentiation factor 88 is up-regulated in epileptic brain and contributes to experimental seizures in rats. Exp Neurol 2017;295:23-35.

31. Wen X, Han XR, Wang YJ, Wang S, Shen M, Zhang ZF, Fan SH, Shan Q, Wang L, Li MQ, Hu B, Sun CH, Wu DM, Lu J, Zheng YL. MicroRNA-421 suppresses the apoptosis and autophagy of hippocampal neurons in epilepsy mice model by inhibition of the TLR/ MYD88 pathway. J Cell Physiol 2018;233:7022-7034.

32. Shibley H, Smith BN. Pilocarpine-induced status epilepticus results in mossy fiber sprouting and spontaneous seizures in C57BL/6 and CD-1 mice. Epilepsy Res 2002;49: 109-120.

33. Paxinos G, Franklin K. Paxinos and Franklin's the mouse brain in stereotaxic coordinates, fourth edition. Mouse brain atlas. Academic, 2012. Pages: 360. ISBN: 978-0123910578.

34. Alyu F, Dikmen M. Inflammatory aspects of epileptogenesis: contribution of molecular inflammatory mechanisms. Acta Neuropsyhiatrica, 2017,29(1):1-16.

35. Bernardino L, Xapelli S, Silva AP, Jakobsen B, Poulsen FR, Oliveira CR, Vezzani A, Malva JO, Zimmer J. Modulator effects of interleukin- $1 \beta$ and tumor necrosis factor- $\alpha$ on AMPA-induced excitotoxicity in mouse organotypic hippocampal slice cultures. J Neurosci 2005;25: 6734-6744.
36. Xu H, Ou F, Wang P, Naren M, Tu D, Zheng R. High dosage of agmatine alleviates pentylenetetrazole-induced chronic seizures in rats possibly by exerting an anticonvulsive effect. Exp Ther Med 2014;8: 73-78.

37. Borges K, Gearing M, McDermott D L, Smith AB, Almonte AG, Wainer BH, Dingledine R. Neuronal and glial pathological changes during epileptogenesis in the mouse pilocarpine model. Exp Neurol 2003;182: 21-34.

38. Mantell LL, Parrish WR, Ulloa L. Hmgb-1 as a therapeutic target for infectious and inflammatory disorders. Shock 2006;25: 4-11.

39. Qiu J, Nishimura M, Wang Y, Sims JR, Qiu S, Savitz SI, Moskowitz MA. Early release of HMGB-1 from neurons after the onset of brain ischemia. J Cereb Blood Flow Metab 2008;28: 927-938.

40. Rasley A, Tranguch SL, Rati DM, Marriott I. Murine glia express the immunosuppressive cytokine, interleukin-10, following exposure to Borrelia burgdorferi or Neisseria meningitidis. Glia 2006;53: 583-592.

41. Wetherington J, Serrano G, Dingledine R. Astrocytes in the epileptic brain. Neuron 2008;58: 168-178.

42. Culcasi M, Lafon-Cazal M, Pietri S, Bockaert J. Glutamate receptors induce a burst of superoxide via activation of nitric oxide synthase in arginine-depleted neurons. J Biol Chem, 1994;269: 12589-12593.

43. Quirie A, Demougeot C, Bertrand N, Mossiat C, Garnier P, Marie C, Prigent-Tessier A. Effect of stroke on arginase expression and localization in the rat brain. Eur J Neurosci 2013;37: 1193-1202.

44. Ahn M, Yang W, Kim H, Jin JK, Moon C, Shin T. Immunohistochemical study of arginase-1 in the spinal cords of Lewis rats with experimental autoimmune encephalomyelitis. Brain Res 2012;1453: 77-86.

45. Drouin-Ouellet J, LeBel M, Filali M, Cicchetti F. MyD88 deficiency results in both cognitive and motor impairments in mice. Brain Behav Immun 2012;26: 880-885.

46. Lim JE, Song M, Jin J, Kou J, Pattanayak A, Lalonde R, Fukuchi KI. The effects of MyD88 deficiency on exploratory activity, anxiety, motor coordination, and spatial learning in C57BL/6 and APPswe/PS1dE9 mice. Behav Brain Res 2012;227: 36-42.

47. Everard A, Geurts L, Caesar R, Van Hul, M, Matamoros S, Duparc T, Denis R, Cochez P, Pierard F, Castel J,Bindels LB, Plovier H, Robine S, Muccioli GG, Renauld JC, Dumoutier L, Delzenne NM, Luquet S, Bindels LB. Intestinal epithelial MyD88 is a sensor switching host metabolism towards obesity according to nutritional status. Nat Commun 2014;5.

48. Kirkman NJ, Libbey JE, Wilcox KS, White HS, Fujinami RS. Innate but not adaptive immune responses contribute to behavioral seizures following viral infection. Epilepsia 2010;51(3):454-64.

49. Ghasemi M, Schachter SC. The NMDA receptor complex as a therapeutic target in epilepsy: a review. Epilepsy Behav 2011;22: 617-640.

50. Zeiler FA, Teitelbaum J, Gillman LM, West M. NMDA antagonists for refractory seizures. Neurocrit Care 2014;20: 502-513.

51. Lopes MW, Soares FMS, de Mello N, Nunes JC, Cajado AG, de Brito D, de Cordova FM, da Cunha RMS, Walz R, Leal RB. Timedependent modulation of AMPA receptor phosphorylation and mRNA expression of NMDA receptors and glial glutamate transporters in the rat hippocampus and cerebral cortex in a pilocarpine model of epilepsy. Exp Brain Res 2013;226: 153-163.

52. Peng WF, Ding J, Li X, Fan F, Zhang QQ, Wang X. N-methyl-daspartate receptor NR2B subunit involved in depression-like behaviours in lithium chloride-pilocarpine chronic rat epilepsy model. Epilepsy Res 2016;119: 77-85.

53. Zheng H, Tang R, Yao Y, JiZ, Cao Y, LiuZ, Peng F, Wang W, Can D, Xing $\mathrm{H}, \mathrm{Bu} \mathrm{G}, \mathrm{Xu} \mathrm{H}$, Zhang Y, Zheng W. MiR-219 protects against seizure in the kainic acid model of epilepsy. Mol Neurobiol 2016;53: 1-7.

54. Golde S, Chandran S, Brown GC, Compston A. Different pathways for iNOS-mediated toxicity in vitro dependent on neuronal maturation and NMDA receptor expression. J Neurochem 2002;82: 269-282. 
55. Lecanu L, Verrecchia C, Margaill I, Boulu RG, Plotkine M. iNOS contribution to the NMDA-induced excitotoxic lesion in the rat striatum. Brit J Pharmacol 1998;125: 584-590.

56. Pérez-Asensio FJ, Hurtado O, Burguete MC, Moro MA, Salom JB, Lizasoain I, Torregrosa G, Lezab JC, Alborcha E, Knowles RG, Lorenzob P. Inhibition of iNOS activity by $1400 \mathrm{~W}$ decreases glutamate release and ameliorates stroke outcome after experimental ischemia. Neurobiol Dis 2005; 18 : 375-384.

57. Coulter DA, Eid T. Astrocytic regulation of glutamate homeostasis in epilepsy. Glia 2012;60: 1215-1226.

58. Petr GT, Sun Y, Frederick NM, Zhou Y, Dhamne SC, Hameed MQ, Clive Miranda C, Bedoya EA, Fischer KD, Armsen W, Wang J, Danbolt NC, Rotenberg A, Aoki CJ, Rosenberg PA. Conditional deletion of the glutamate transporter GLT-1 reveals that astrocytic GLT-1 protects against fatal epilepsy while neuronal GLT-1 contributes significantly to glutamate uptake into synaptosomes. J Neurosci 2015;35: 5187-5201.

59. Liu XJ, Liu T, Chen G, Wang B, Yu XL, Yin C, Ji RR. TLR signaling adaptor protein MyD88 in primary sensory neurons contributes to persistent inflammatory and neuropathic pain and neuroinflammation. Sci Rep 2016;6.

60. Shen Y, Qin H, Chen J, Mou L, He Y, Yan Y, Zhou H, Lv Y, Chen Z, Wang J, Zhou YD. Postnatal activation of TLR4 in astrocytes promotes excitatory synaptogenesis in hippocampal neurons. J Cell Biol 2016;215(5):719-734. 